$$
\begin{aligned}
& \text { 震 } \\
& \text { 步仿 }
\end{aligned}
$$

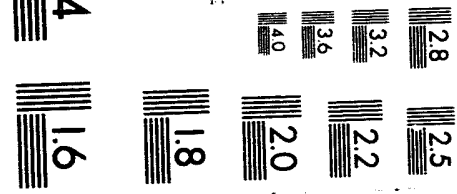



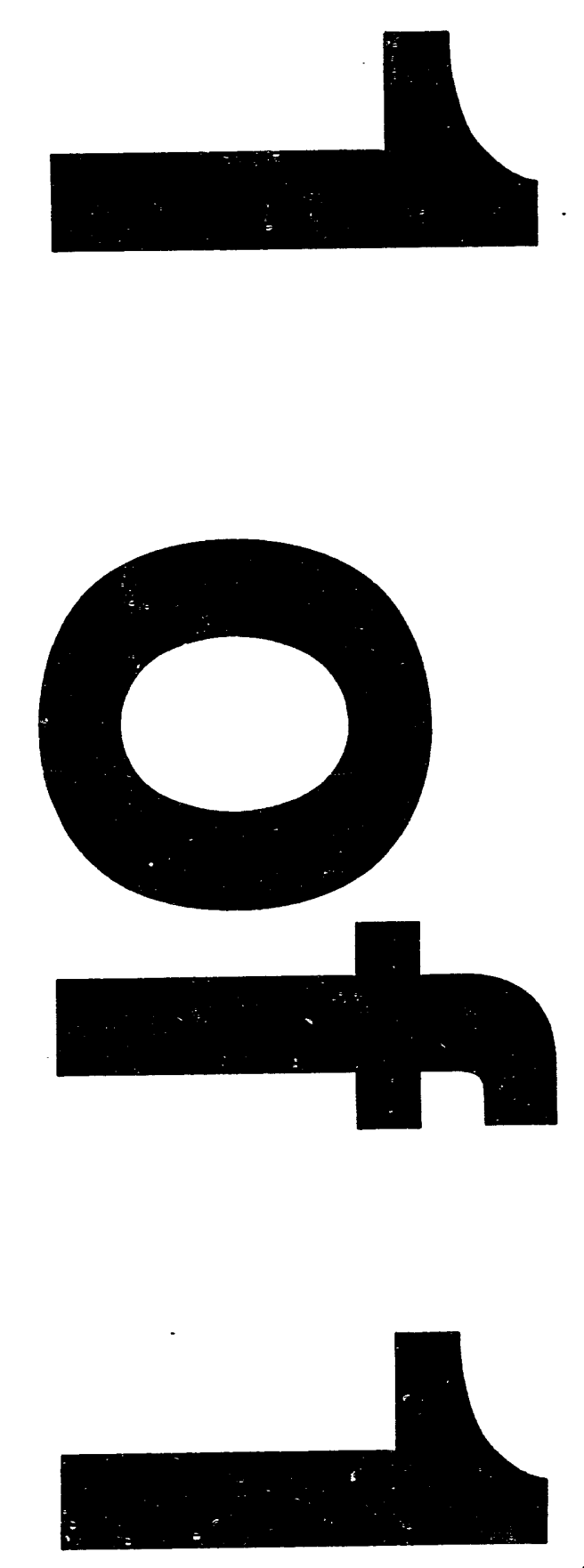
UCRL-LR-115636

Distribution Category UC-705

\title{
An Investigation of DUA Caching Strategies for Public Key Certificates
}

\author{
Terry Ching Cheung
}

Manuscript date: November 1993

LAWRENCE LIVERMORE NATIONAL LABORATORY University of California • Livermore, California • 94551

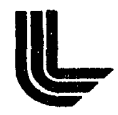




\title{
An Investigation of DUA Caching Strategies for Public Key Certificates
}

\author{
By
}

\section{Terry Ching Cheung \\ B.S. (University of California, Berkeley) 1987}

\section{THESIS}

Submitted in partial satisfaction of the requirements for the degree of

\section{MASTER OF SCIENCE}

in

\author{
Computer Science \\ in the \\ GRADUATE DIVISION \\ of the \\ UNIVERSITY OF CALIFORNIA \\ DAVIS
}

Approved:

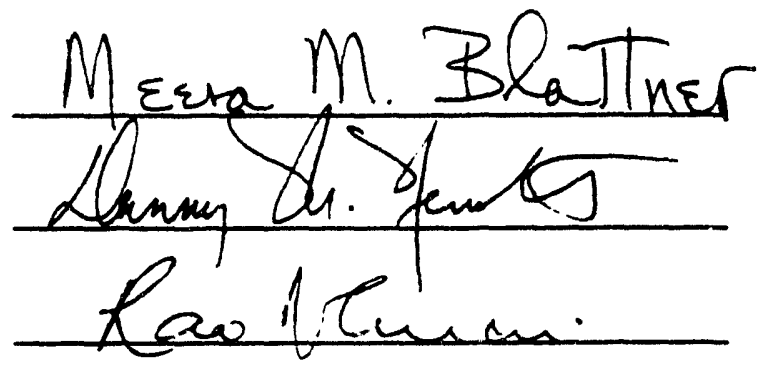

Committee in Charge 


\section{Acknowledgements}

I would like to express my gratitude to members of my thesis committee: Professor Meera Blattner, Professor Venkateswara Vemuri, and Dr. Dan Nessett.

I would like to thank Dr. Dan Nessett again for ideas, guidance, editing, and feedback throughout the project. 


\begin{abstract}
Internet Privacy Enhanced Mail (PEM) provides security services to users of Internet electronic mail. PEM is designed with the intention that it will eventually obtain public key certificates from the X.500 directory service. However, such a capability is not present in most PEM implementations today. While the prevalent PEM implementation uses a public key certificate-based strategy, certificates are mostly distributed via e-mail exchanges, which raises several security and performance issues.
\end{abstract}

In this thesis research, we changed the reference PEM implementation to make use of the X.500 directory service instead of local databases for public key certificate management. The thesis discusses some problems with using the X.500 directory service, explores the relevant issues, and develops an approach to address them. The approach makes use of a memory cache to store public key certificates. We implemented a centralized cache server and addressed the denial-of-service security problem that is present in the server. In designing the cache, we investigated several cache management strategies.

One result of our study is that the use of a cache significantly improves performance. Our research also indicates that security incurs extra performance cost. Different cache replacement algorithms do not seem to yield significant performance differences, while delaying dirty-writes to the backing store does improve performance over immediate writes. 
Keywords:

PEM, X.500, directory, public key certificate, CRL, validation, memory cache, cache replacement algorithms. 


\section{Table Of Contents}

1. Introduction........................................................................... 1

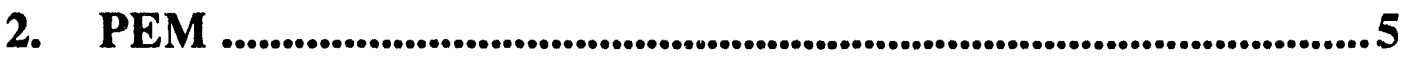

3. The Problem ...................................................................................... 16

4. Analysis .................................................................................................................. 19

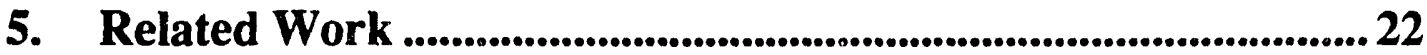

6. Implementation ........................................................................24

7. Cache replacement algorithms ...........................................................29

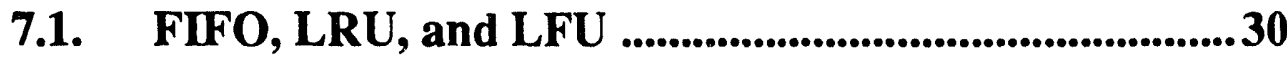

7.2. New replacement algorithm (DEPTH) ........................... 30

8. Performance Results.........................................................................39

8.1. No Validation ........................................................................49

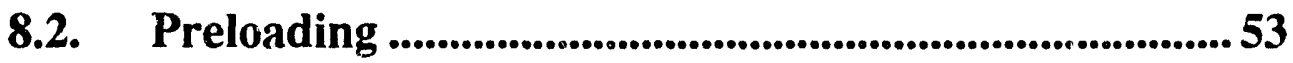

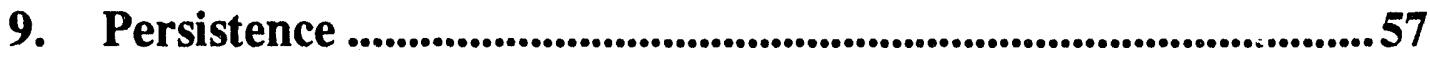

10. Conclusions and Future Work ....................................................6 60

References ...................................................................................................662

Appendix A: Flow Diagrams for the Cache Server Operations .....64

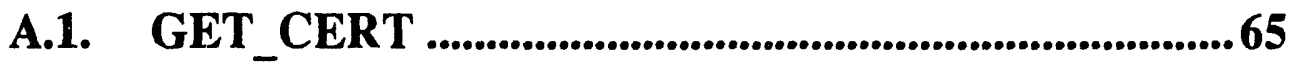

A.2. PUT_CERT ........................................................................66

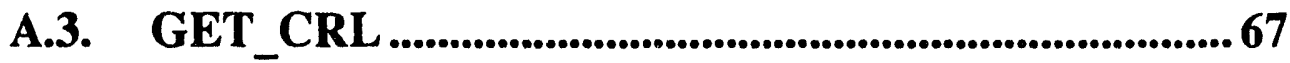

A.4. PUT_CRL ........................................................................... 68

A.5. Storing a certificate or CRL into the caches................69 


\section{Introduction}

Computers play a very important role in people's lives today. One of the common uses of computers is to exchange messages electronically among users. However, with the number of cases of computer abuse on the rise, and the fact that electronic messages can be intercepted, destroyed, replayed, etc., simple e-mail exchanges will not be sufficient for many computer applications.

The Internet consists of the largest group of computer users. The multiprotocol open internet already includes networks in over 127 countries. The network, which includes Bitnet, FidoNet, IP, UUCP, and OSI, connects over 1.5 million computers with over 10 million users [Ishi93]. Internet Privacy Enhanced Mail (PEM) is envisioned to be the chief secure mail exchange technology in the Internet.

PEM is a standard for providing security services to message exchanges in the Internet. There are four security services: message integrity, authentication, confidentiality, and non-repudiation of origin. Message integrity guarantees that a message has not been exposed to accidental or malicious alteration or destruction. Authentication allows a receiver to correctly identify or verify the originator of a message. Confidentiality prevents disclosure of a message to an unauthorized party. Non-repudiation of origin prevents the originator of a message from denying having sent the message. The security services are provided by applying cryptographic measures (e.g. encryption) to messages transferred between end systems by the Message Transfer System. The PEM protocols are currently specified in [RFC1421, RFC1422, RFC1423, RFC1424]. 
In a conventional telephone system, people can discover information about a user or an organization by searching for the user name in the white pages of a telephone book. The equivalent service in the computer world is the X.500 directory service. The X.500 directory service as specified in CCITT X.500 Recommendations for directory services [X.500-88] is a collection of open systems which cooperate to hold a logical database of information about a set of objects in the real world. The X.500 directory service works in a similar fashion as the white pages service in the conventional telephone system in that it allows users to look up information on an entity (e.g. a user, an organization, etc.). The X.500 directory service, however, typically provides much more information in addition to that provided by the conventional telephone system. The X.500 directory service typically provides information on a person's name, address, telephone number, fax number, etc. Users (including both people and computer programs) who wish to access or modify information held in the directory do so through a Directory User Agent (DUA). DUAs are application entities (i.e. processes) that act on behalf of users in communication with Directory System Agents (DSAs), distributed servers that manage and hold the information for the X.500 directory.

Transfer of information between DUAs and DSAs are subjected to a number of potential threats, just like any other types of electronic transmissions. To counter the threats, a standard, termed the X.509 directory authentication framework [X.509-88], has been developed. The X.509 directory authentication framework specifies requirements for two types of authentication exchange: simple authentication and strong authentication. Simple authentication uses conventional password checking to authenticate a user, while strong authentication requires the use of public key certificates. Each public key certificate is data that contains the public key (see the following paragraph for more information on pubic keys) and the name of a user, and guarantees the correctness of the association between the public key and the name. 
Public key certificates are becoming common in situations where higher levels of security are required in distributed environments.

In cryptographic security, the transformation of plain text into crypto text is called encryption. The reverse process, the transformation of crypto text into plain text is called decryption. The transformations are controlled by a key. If the same key is used for both encryption and decryption, the cryptographic system is symmetric. If different but mathematically related keys are used for encryption and decryption, the cryptographic system is called asymmetric. These are the only two cryptographic systems in common use today. Asymmetric cryptographic systems are also called public-key systems [Carr87]. In public-key systems, the public key of a user is made available to everyone, while the private key is kept secret by the user. Both keys can be used to encrypt or decrypt messages. That is, if the public key of a user is used to encrypt a message, the corresponding private key must be used to decrypt the message, and vice versa. If user $A$ wants to send an encrypted message to user B, A uses B's public key to encrypt the message and when B receives the message, he/she decrypts it using his/her own private key. Only B will be able to read the message since he/she is the only one who possesses the private key. If A wants to send a digitally signed message (for authentication), he/she encrypts the message with his/her own private key and then sends the message. The receiver of the signed message can decrypt the message using A's public key (supposedly obtainable from a publicly available service, e.g. the X.500 directory service). If the decrypted message is readable, the receiver will know for sure that the message came from A, since only A can sign a message that is decryptable with A's public key.

PEM uses both types of cryptographic systems and supports both symmetric and asymmetric key distribution. However, the prevalent implementation for key 
distribution uses a public key certificate-based strategy, modeled after the X.509 directory authentication framework. This scheme provides an infrastructure compatible with the X.509 framework. According to [RFC1422], public key certificates can be stored in directory servers, transmitted via non-secure message exchanges, or distributed via other means. Since directory servers are not widely available, most of the existing PEM implementations have chosen to send and receive certificates in PEM messages and keep them in a local database rather than obtaining them from X.500 directory servers.

However, storing certificates locally poses some problems, the most serious of which is the limited availability of certificates to PEM users. This thesis delineates this problem and proposes a solution. Directory servers are used to store certificates and a centralized certificate server is used to interact with directory servers on behalf of PEM users. The use of a centralized server raises at least one security issue. In particular, the centralized server is vulnerable to denial-of-service attacks. Denial-of-service attacks can make the server crash or perform extra work that makes it unavailable. This security issue is examined and addressed. The performance of the centralized certificate server is examined by measuring the response times of requests to the server. Various memory caching techniques are examined in order to discover the best management strategy. 


\section{PEM}

Privacy Enhanced Mail (PEM) is a suite of algorithms, data definitions, and protocols that in concert protect electronic mail from various security and privacy threats [RFC1421, RFC1422, RFC1423, RFC1424]. PEM provides four security services: authenticity, integrity, non-repudiation of origin, and confidentiality. A PEM message contains two parts - the message header and the message content. The message header contains information that is used by PEM during the processing of the message. The fields of a PEM message are "Proc-Type," "Content-Domain," "Originator-Certificate," "Issuer-Certificate," "Originator-ID-Asymmetric," and "MIC-Info."

The "Proc-Type" field identifies the type of processing performed on the message. There are three possible types: MIC-CLEAR, MIC-ONLY, and ENCRYPTED. A message integrity code (MIC) is a value calculated using a one-way hash function on the message. The MIC is protected by encrypting it with the private key of the originator. The encrypted MIC value forms a digital signature over the PEM message body. The recipient of a message first decrypts the encrypted MIC value using the originator's public key, and then verifies the MIC by performing the hash function on the message and comparing it with the decrypted MIC. Thus, the MIC is used in both integrity and authentication checks. A MIC-CLEAR message calculates the MIC on the message, but does nothing to the message content so that non-PEM users can also read PEM messages. A MIC-ONLY message indicates that the message has an additional encoding performed on it, to ensure that the transmission of the message through different underlying messaging systems does not automatically invalidate the integrity and authentication checks. 
An ENCRYPTED message provides confidentiality, in addition to the services already provided by MIC-ONLY messages. The message content is encrypted using symmetric cryptography. The symmetric key used to encrypt the message is used exclusively for the message. This message encipherment key is then encrypted using the public key of the recipient. Thus, only the recipient is able to obtain the message encipherment key and to view the message content.

The "Content-Domain" field indicates the type of standard representation the original message content will be transformed into. The value "RFC822" indicates that the message is to be processed as would RFC-822 mail. RFC-822 is a standard used to transmit messages in the Internet environment.

The "Originator-ID-Asymmetric" field contains information regarding the identity of the originator. Is is used when the originator's public key certificate is not transmitted in the message.

The "MIC-Info" field indicates the one-way hash-function used in generating the MIC. In the examples shown, the function is RSA-MD5. In addition, it indicates the method used to protect the MIC, which, in the examples given, is the RSA algorithm. RSA is the most commonly used public key cryptographic algorithm.

[RFC1422] requires PEM to provide the capability of including the originator's certification path in the message header. The certification path of a user is used in checking the validity of the user's certificate, and will be explained in detail below. This information appears in the "Originator-Certificate" and "Issuer-Certificate" fields. An example of a message that contains the certification path of the originator is shown below in Figure 2.0, while one without the certification path is shown in Figure 2.1: 
----BEGIN PRIVACY-ENHANCED MESSAGE-----

Proc-Type: 4,MIC-CLEAR

Content-Domain: RFC822

Originator-Certificate:

MIICBjCCAW8CAQIwDQYJKoZIhvcNAQECBQAwUzELMAkGA1UE BhMCVVMxCzAJBgNVBAgTAk1EMSQwIgYDVQQKExtUcnVzdGVk IEluZm9ybWF0aW9uIFN5c3RlbXMxETAPBgNVBAsTCEdsZW53b29k MB4XDTkzMDUyOTA0MTYxOVoXDTk1MDUyOTA0MTYxOVowbT ELMAkGA1UEBhMCVVMxCzAJBgNVBAgTAk1EMSQwIgYDVQQK ExtUcnVzdGVkIEluZm9ybWF0aW9uIFN5c3RlbXMxETAPBgNVBAsT CEdsZW53b29kMRgwFgYDVQQDEw9KYW1lcyBNLiBHYWx2aW4w dzAKBgRVCAEBAgIDAANpADBmAmEA3faBc3P78SBC43fo+avW/N VrP9ibbe/Hit7oW2wiil wZ/QiJHGi91AvfrDFtbJUmmqR2txnb30lb2TzscZ V/gnreZKtwLox3/RzBRQeNNZv2RtC9yMJyGtsXJNMGgdrfAgEDMA0 GCSqGSIb3DQEBAgUAA4GBADFG28/27CTw8dKR11+Y2//MlVVpH of +y9kxLK9Sebi91HfKStNSBq4nn3lubbGQVg0d2kW9UgDmvUS06lne 2IWWYMXwdXVrTZz7w+MAclBjrWhwlikv93GUz4Ezs2WE5bzbFom gbVUDGqCZkx2cqYfuBGYJQ31KOw1s3UhDb+OK

Issuer-Certificate:

MIICATCCAWoCAQIwDQYJKoZIhvcNAQECBQAwRDELMAkGA1 UEBhMCVVMxCzAJBgNVBAgTAk1EMSgwJgYDVQQKEx9UcnVzd GVkIEluZm9ybWF0aW9uIFN5c3RlbXMgUENBMB4XDTkzMDUyOT AwMDc0MloXDTk1MDUyOTAwMDc0MlowUzELMAkGA1UEBhMC VVMxCzAJBgNVBAgTAk1EMSQwIgYDVQQKExtUcnVzdGVkIEluZ m9ybWF0aW9uIFN5c3RlbXMxETAPBgNVBAsTCEdsZW53b29kMIG aMAOGBFUIAQECAgQAA4GLADCBhwKBgQC3apEqJMFVbnhy3op 4d6NqbQox23npkJtPInbf4NQi4/ZmE8moym3ZlatSkpkKQeaQ1IPDeJS YQUsp2SRxWHE2GfbBcIn9obDS1B7i/F8RE1ObTGn/euNced96sRRN vre5aPqt6NGWkwTHHkiK8/Wwz/EVqBgVtaNID4vAWa6hXwIBAzA NBgkqhkiG9w0BAQIFAAOBgQBov/le/jio0yZFplZGhWeNtlgCawg3as Z×3//YbSPUobF08GOZsFD6DvSIRFTAi3T66iRMHbRE6CPYIuObCD 2 wQAvrU5siNm3fCM70x/qZf6n2L9k91IvsfLKx84frojtoKS9IZj28GLII fw94Ag1bmcRhRPhBsRxhJ4wGZGMq9g==

Figure 2.0 An incoming PEM message with the certification path of the originator included (continue on next page) 
Issuer-Certificate:

MIIB8jCCAVsCAQEwDQYJKoZIhvcNAQECBQAwRDELMAkGA1U EBhMCVVMxCzAJBgNVBAgTAk1EMSgwJgYDVQQKEx9UcnVzdG VkIEluZm9ybWF0aW9uIFN5c3RlbXMgUENBMB4XDTkzMDUyODE 3MTEyN1oXDTk1MDUyODE3MTEyN1owRDELMAkGA1UEBhMC VVMxCzAJBgNVBAgTAk1EMSgwJgYDVQQKEx9UcnVzdGVkIEluZ m9ybWF0aW9uIFN5c3RlbXMgUENBMIGaMAoGBFUIAQECAgQAA 4GLADCBhwKBgQDbLxaRIS3u54yyRgVDI5dcE9nlasL8fJqOGlyo7xH 2FZnr3kUfsFj70GiYsr6UbvqwKnyfMIRUrXDUa64leGmft3SK27psDU HOynRSCc40d/HrDf810U5tnTamBKUIMqivK4GoLOtMRA leX6hALA vLLgK1HbnwZAo6GqQGW8CIJQIBAzANBgkqhkiG9w0BAQIFAAOB gQDBp5aC6oV6IuFi8JCctq57bew604HHNllgijp7zdXafq6jctRg2g91k/y FWh19bJC/tNrb0WVwuZOs5L/FToPMNIIHzaW/YSROBmyhTDYaKH ZGj0P1+iNjMbHt9dm1QEHGIfKgBwFidItnOa74DfkXdijlPRnr/+E2Ib6 $\mathrm{PM}+\mathrm{hEfQ}==$

MIC-Info: RSA-MD5, RSA, wvmjSBMkRp1WiXp2BOj9om+ahxFzmiwivm0xrFZDBMcjL3f2rCiwr VpCjKKydTHbMvORULGPK5yPD+o910KZPxXLaMjTem9csAGvgln 5O2gnZXyOdPAMdnMBaZoUugBi

[ASCII text message]

-----END PRIVACY-ENHANCED MESSAGE--.--

Figure 2.0 An incoming PEM message with the certification path of the originator included (continue from previous page)

----BEGIN PRIVACY-ENHANCED MESSAGE-----

Proc-Type: 4,MIC-CLEAR

Content-Domain: RFC822

Originator-ID-Asymmetric: MFMxCzAJBgNVBAYTAIVTMQswCQYDVQQIEwJNRDEkMCIGA1 UEChMbVHJ1c3RIZCBJbmZvcm1hdGlvbiBTeXN0ZW1zMREwDwYD VQQLEwhHbGVud29vZA==,02

MIC-Info: RSA-MD5, RSA, BpCu5i/vNJFNX64bj4KuRr8Jm05gdfjIIO5WQaSTXAGkx09ivq97Gtmd ksgshOkdqynllxTSph0s6DtNN5girn2Q/u08q44XLYbk6vYxA9g37w/L11 eqw7CldPLPOtQT

[ASCII text message]

-.---END PRIVACY-ENHANCED MESSAGE-----

Figure 2.1 An incoming PEM message without the certification path of the originator 
The message in Figure 2.0 contains three fields in addition to those shown in Figure 2.1. The "Originator-Certificate" field contains the certificate of the originator, while the "Issuer-Certificate" fields contain the certificates of the originator's CA and his PCA. Together, these three fields contain the certification path of the originator.

To support its security services, PEM makes use of public key certificates that conform to the X.509 recommendation. An X.509 public key certificate binds a public key to the distinguished name (DN) of an entity and also contains the DN of the entity that vouches for the binding. The entity on whose behalf the certificate is issued is termed a subject, while the entity that vouches for the binding is termed an issuer.

The Distinguished Name (DN) of an entity is the global, authoritative name of the entry in the X.500 directory. Entries in the X.500 directory are named using a hierarchical structure termed the Directory Information Tree (DIT). This structure is used to uniquely name entries [Rose92]. A simple DIT is shown below:

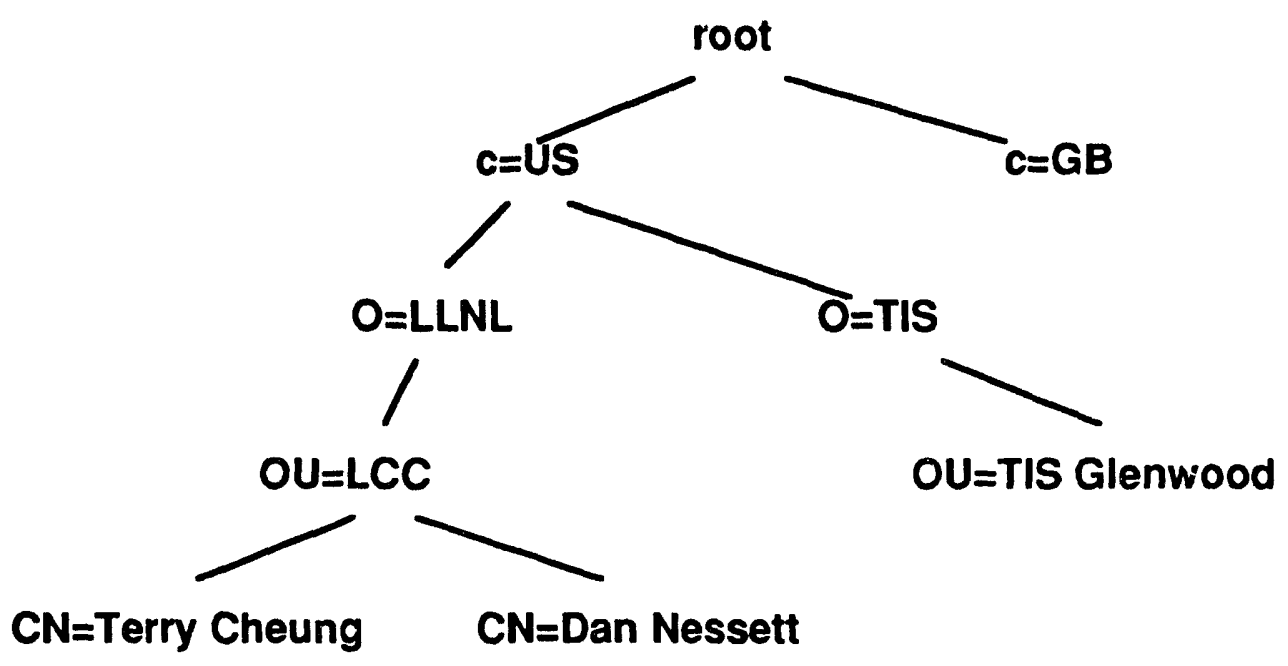

Figure 2.2 A simple DIT 
The DN of each entry is formed by tracing the path from the root of the tree to the entry. The root of the entry is not part of the DN. For example, the DN for the left most leaf in the simple tree in Figure 2.2 in short form is $\{C=U S, O=L L N L, O U=L C C$, $\mathrm{CN}=$ Terry Cheung . A more detailed description for the $\mathrm{DN}$ is:

countryName (C) is "US"

organizationName $(\mathrm{O})$ is "Lawrence Livermore National Laboratory" organizationalUnitName (OU) is "Livermore Computation Center" commonName $(\mathrm{CN})$ is "Terry Cheung"

where countryName, organizationName, etc. are string representations of standard attribute types defined in [X.500-88] and "US", "Lawrence Livermore National Laboratory" are string representations of the corresponding attribute values.

An X.509 public key certificate contains the following information: version - the version of the certificate format used in this certificate serial number - a number assigned by the issuer of this certificate that is unique among all the certificates issued by the issuer signature algorithm - the algorithm used by the issuer to create the digital signature for this certificate issuer - the entity that vouches for the binding between the public key and the subject entity

validity period - the time interval during which the certificate is valid subject - the subject entity for whom the certificate is issued subject public key info - the public key, along with the algorithm that generates the key signature - the digital signature created using the issuer's public key

A sample X.509 certificate used in PEM contains the following values: 


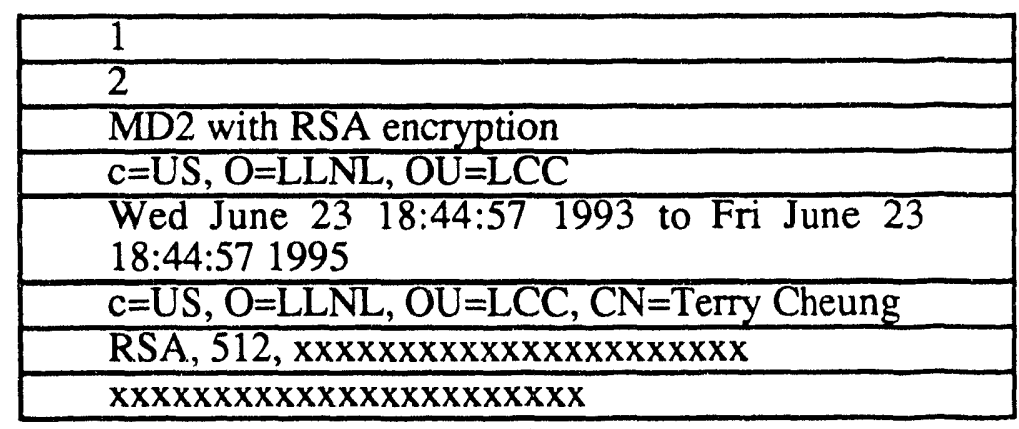

Figure 2.3 A sample public key certificate

where the xxx's in the second to last entry represent the data bits in the public key and the $x_{x x}$ 's in the last entry represent the data bits in the digital signature. In the example certificate, MD2 in concert with RSA is the algorithm used to generate the digital signature. RSA requires a parameter, which has a value of 512 in the sample certificate.

In order to prevent forgery of public key certificates and to guarantee their integrity, public key certificates must be validated. To validate certificates, a certification system must be established. The Internet community has established a certification system that takes the form of a single rooted tree. Kent provides a description of this certification system in [Kent93]:

"The root of this tree is designated the Internet PCA Registration Authority (IPRA) and it is operated under the auspices of the Internet Society, a nonprofit, professional organization that promotes use of Internet technology on a world wide basis. The IPRA provides a reference point from which all certificates in this certification hierarchy can be validated. The IPRA establishes a common policy that applies to all certificates issued under this hierarchy. The IPRA directly issues certificates to a second tier of entities designated Policy Certification Authorities (PCAs) which, in turn, issue certificates to CAs. CAs issue certificates to (subordinate) CAs or directly users (individuals, organizations, organizational roles, mailing lists)." 
A CA vouches for the binding between its immediate subordinate CAs' or users' distinguished names (DNs) and their public keys within an organization or organizational unit. The CA's DN is that of the organization or organizational unit and the first part of an immediate subordinate entity's DN is that of the CA's DN and the last element is something that uniquely identifies the entity (e.g. the organization unit's name or the user's name).

A PCA vouches for the binding between a CA's DN and its public key. A PCA is typically run by a well-known organization. The PCA issues certificates for Certification Authorities run by other organizations. By using a particular PCA, messages from an organization are verified by following the certification path (to be explained below) to the IPRA certificate without having to retrieve the message originator's key using secure, out-of-band means.

A sample certification hierarchy for the Internet community is shown in Figure 2.4 , where the IPRA is at the root of the hierarchy. A PCA operating by Trusted Information Systems (TIS) is immediately below the IPRA. The TIS PCA issues CA certificates for its members, Lawrence Livermore National Laboratory (LLNL), Massachusetts Institute of Technology (MIT), and TIS division at Glenwood, Maryland. LLNL in turn issues a CA certificate for its organizational unit Livermore Computation Center (LCC), and LCC issues a certificate for the employee Terry Cheung. 


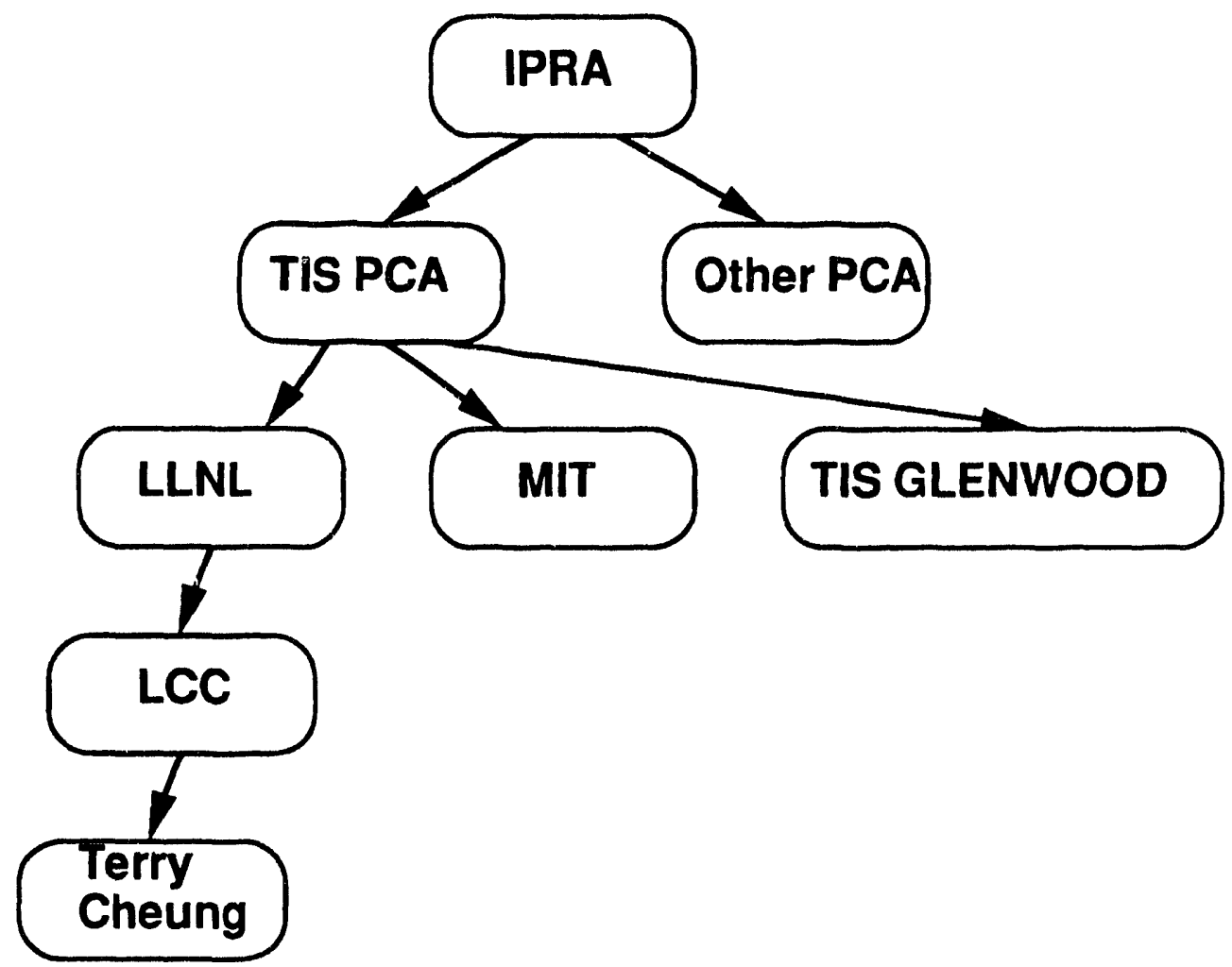

Figure 2.4 Sample Internet certification system

A certification path is an ordered sequence of certificates. The first element of a certification path is the certificate for a PCA, and every element of the certification path except the last one is a certificate for a certification authority that issues the next one. For example, the certification path for LCC in Figure 2.4 is an ordered sequence of certificates for (TISPCA, LLNL, LCC \} and that for Terry Cheung is (TISPCA, LLNL, LCC, Terry Cheung\}. The IPRA certificate is not included in the certification path because it is a well-publicized certificate and a PEM implementation must have obtained it through some out-of-band means.

To ensure that the DNs of all the entities are unique, there is a subordination rule on the naming of CAs. The DN of the subject in the certificate issued by a CA must be subordinate to the DN of the CA. The only exception to this rule is that of the CA 
certificates issued directly by PCAs. For example, the DN of the subject for LLNL certificate is $\{C=U S, O=L L N L\}$. The $D N$ of the subject for $L C C$ is $\{C=U S, O=L L N L$, $\mathrm{OU}=\mathrm{LCC}$ ), which is subordinate to $\{\mathrm{C}=\mathrm{US}, \mathrm{LLNL}\}$ (Figure 2.2).

With the establishment of a certification system, the validation of a certificate as specified in [RFC1422] involves the following four procedures. First, since the digital signature of a certificate is generated using the private key of its issuer, the public key of its issuer is required in order to perform the decryption so the validity of the signature can be checked. Secondly, each certificate has a lifetime associated with it, indicated by the validity interval included in the certificate. The validity interval is checked to see if the certificate has expired. Thirdly, an RFC compliant PEM implementation checks to see if the issuer DN and the subject DN satisfy the DN subordination requirement.

Finally, a certificate can be invalidated before its expiration time, by way of revocation. Certificate Revocation Lists (CRLs) are the data structures employed to perform revocations. The certificate (or more specifically the serial number of the certificate) is checked against its issuer's CRL to see if it has been revoked.

[RFC1422] defines CRLs for use in strong authentication in the X.500 directory. However, current X.509 CRLs are missing some vital information. Hence, [RFC1422] defines a CRL syntax that is more suitable for use in PEM.

The PEM CRLs contain the following information:

signature algorithm - the algorithm used by the issuer to generate the digital signature of this $\mathrm{CRL}$ issuer - the entity that vouched for the certificates in the list of certificates to be revoked 
last update - time of last update of this CRL

next update - time when next update of this CRL is to occur; if the

current time exceeds the time containing in this field, the CRL

is no longer valid

revoked certificates - list of certificates revoked; each entry of the list

contains the serial number of the certificate revoked, and the time

when the revocation becomes effective

A sample CRL used in PEM contains the following information:

MD2 with RSA encryption $\mathrm{c}=\mathrm{US}, \mathrm{O}=\mathrm{LLNL}, \overline{\mathrm{OU}}=\mathrm{LCC}$

Wed July $2118: 45: 361993$

Wed July 28 18:45:36 1993

12, Thu March 19 13:20:03 1993

258, Mon May 17 15:47:06 1993

528, Wed June 2 09:43:25 1993

Figure 2.5 A sample CRL

When a certificate is to be checked for revocation, its issuer's CRL is located. A certificate's issuer's CRL is the CRL with the same issuer DN as that of the certificate. The serial number of the certificate is then compared against the serial numbers in the list of revoked certificates. If a match is found, the certificate is considered invalid.

CRLs are also intended to be managed in the X.500 directory. In the absence of a global directory structure today, CRLs are mostly cached in local databases by PEM. The IPRA requires every PCA to maintain a database of CRLs of all the CAs under the PCA sub-hierarchy. PEM users must contact the PCAs in order to obtain CRLs. 


\section{The Problem}

Most PEM implementations currently use a push-model for certificate proliferation. That is, certificates are transmitted via non-secure message exchanges. The certificates are pushed (attached) onto messages by the PEM implementation at the sender site. The PEM implementation at the receiving site removes them and stores them in a local database. Under this model, a certificate for user A at one site is available to user B at a different site only if the certificate for user A is included in a mail message received either on user B's machine or on a machine sharing its certificate database with user B's machine. Sharing of certificate databases is possible using a distributed file service such as NFS.

The push-model has two serious drawbacks. For those implementations not using a distributed file service, a certificate stored in the local database at one site is unavailable to users at another site. This means the sender of a message must either include a full (or partial if the PEM User Agent does some optimization) certification path every time he sends a message, resulting in performance degradation and waste of bandwidth, or have confidence that his full certification path is available at a receiving site in order for the message to be processed by PEM. Furthermore, a sender cannot send an encrypted message if the receiver's certificate is not in the sender's database, since the receiver's public key is needed to create the encrypted message. This implies that some clear-text message exchanges must take place before the confidential exchanges start, creating a bootstrap problem.

The second drawback occurs when a distributed file service is used to alleviate the above problem. Sharing certificate databases requires the existence of trust 
relationships between the machines using the distributed file service. That is, suppose machine A is the site where a shared certificate database actually is stored. Machine A must trust the sharing sites enough to accept mount requests from them. This trust supersedes that based on PEM considerations, since remotely mounting a file system using a typical distributed file service grants the remote system significant file system privileges. If machine A does not trust another machine B, B cannot share A's database, and furthermore, if A wrongly trusts machine $\mathrm{C}$ and shares its database with $\mathrm{C}$, the integrity of the shared database may be corrupted. While this cannot result in the acceptance of bogus certificates, since all certificates are signed, it can introduce a denial-of-service threat.

One way to solve this problem is for PEM to use a pull-model for certificates. Under this model, certificates are stored in X.500 directory servers and later retrieved by PEM implementations. One problem with this model is that it could seriously impact the performance of the PEM software. Under the pure pull-model, each certificate retrieval/storage operation would ultimately result in at least one directory request. Since directory requests are much slower than accesses to a local database, and the sending/receiving of each message requires one to ten or more certificate retrieval/storage operations, the pull-model could seriously degrade the performance of the PEM software.

Assuming the performance problems of using directory servers are solvable, the problem of transitioning from the push-model to the pull-model still remains. Because directory servers may not be widely available when the PEM implementations using the pull-model are ready for use, the number of certificates that can be retrieved from directory servers would initially be very limited. If the push-model is eliminated completely, a PEM implementation using the pure pull-model cannot operate when the 
certificates it needs are not stored in directory servers. To make a PEM implementation with the pull-model function reasonably, the push-model has to be incorporated into the design, and thus a reasonable PEM implementation would combine both models, rather than using the pull-model alone. 


\section{Analysis}

In changing a PEM implementation from a push-model to a pull-model, there are many issues to address. The first is the availability of certificates to users at different sites. To solve this problem, the obvious solution is for PEM users at different sites to share a database. This increases availability since the certificates that would have otherwise been in different databases would now all be stored in the shared database. We have discussed providing this sharing through the use of a distributed file service and demonstrated that this introduces the necessity of trust relationships that may not exist. Another alternative is to use a centralized server that manages the certificates and accepts certificate retrieval/storage requests from PEM clients.

If PEM is implemented with a straight pull-model approach, that is, if each operation to retrieve/store a certificate ultimately results in one or more requests to the X.500 directory, PEM will suffer significant performance problems. To make the PEM implementation with the pull-model more practical, response time needs to be improved. If the approach of the centralized server is taken, the server can use a memory cache and a long-term backing store to store the certificates, and apply appropriate cache management strategies. Alternatively, [RFC1422] recommends caching certificates locally in the UA. However, in the absence of a ubiquitous directory service, this approach falls back to the current scheme of storing certificates in local databases used by most current PEM implementations, and suffers from the operational difficulties described above.

There are some security considerations when using the approach of a centralized server with a cache. In particular, the server is vulnerable to denial-of-service attacks. If 
a PEM user sends a request to the centralized server to store an invalid certificate and the server stores that certificate into the cache and its backing store without question, invalid certificates could displace valid ones and service could be distupted. The solution to this problem is to perform validation on all certificates before they are stored in the centralized server's cache or backing store. Validation can degrade performance, so the designer should develop an approach that minimizes this degradation.

Since directory servers are not widely available yet, in order to make a smooth transition from the push-model to the pull-model, we need a place to store and retrieve certificates that cannot be placed in the directory hierarchy. One solution is to have the centralized server store them in its local file system on the machine where the server runs. Another alternative is to store them in some designated part of the directory information tree (DIT). This piece of the directory structure must be writable by the centralized server.

A centralized server could be designed either to service one PEM client at a time, or to service multiple clients concurrently. The former implies that the server could be temporarily inaccessible to users when it is busy servicing another client. If so, the PEM software must take appropriate actions, such as either returning an error indication to the PEM user indicating that the server is unavailable, or repeatedly issuing connection requests to the server, giving up after either a certain amount of time or a certain number of retries. A better engineering design allows the server to service multiple clients concurrently since this eliminates many of these resource sharing problems.

Since certificates and CRLs share many common characteristics, CRLs can be managed similarly. However, caching of CRLs brings up a major security issue, that is, PEM clients might not be getting the most recent CRLs that are issued before their next 
scheduled issue date. This could be a problem if non-repudiation of origin is critical to an application. One possible solution to this problem is to provide PEM clients with an option to force the cache server to obtain the most recent CRLs from the directory servers. Another approach is to have a server that will discard CRLs that have been in the cache longer than a specified period of time. Different applications might call for different approaches, or a combination of both approaches might also be more suitable for certain situations. 


\section{Related Work}

Trusted Information Systems has produced a reference implementation of PEM called TIS/PEM [Galv92]. TIS/PEM provides privacy enhancement services to text messages on top of the Rand MH Message Handling System. The database mechanism

used by TIS/PEM stores public key certificates in the UNIX file system. Each certificate in TIS/PEM is stored in the local database as a single UNIX file.

Digital Equipment Corporation has built four PEM prototypes that run on VMS [Tay193]. The last two prototypes have incorporated the use of public key certificates. For the purposes of the prototypes, a centrally generated certificate database was copied to individual systems.

There is an experimental PEM implementation [Hous92] that does not store certificates. It is able to send or receive public key certificates in message headers, but provides only key management and stores public keys in a local key cache.

There are many implementations of the X.500 directory service. These include but are not limited to Alliance OSI X.500 by Touch Communications Inc., DCE/GDS by Open Software Foundations, Inc., DS-520 by Retix, Directory 500 by OSIware Inc., HP X.500 Distributed Directory Software by Hewlett Packard, INTERACTIVE Systems' X.500 DSA/DSAM by INTERACTIVE Systems Corporation, OSI-DSA by Unisys Corporation, UCOM.X 500 by 3Com Corporation, VTT X.500 by Technical Research Centre of Finland, WIN/DS by The Wollongong Group, and QUIPU of the ISODE. Among the Directory implementations, QUIPU is gaining more and more popularity. It 
is an implementation of the OSI directory service contained in ISODE, an openly available implementation of upper-layer OSI protocols [ISOD92].

Cache design is a mature well-understood technology. Smith [Smit82] provides a good overview of the design of cache memories. Some of these concepts are applicable to the design of a memory-based certificate cache. For example, an important aspect in the design of memory caches involves the selection of strategies for storing data to secondary storage. There are two well-known strategies - write-through and write-back (called write-through and copy-back in [Smit82]). In write-through, data written to the cache are immediately transmitted to secondary storage, while in writeback, data written to the cache initially modify the cache, but replaced data are stored to secondary storage at a later time. Another important aspect of cache architecture is the replacement algorithms used. Of the commonly known ones, both FIFO (first in first out) and LRU (least recently used) are common. In addition, LFU (least frequently used) is another well-known cache replacement algorithm. 


\section{Implementation}

We have taken the following approach in our design of a centralized certificate server to support PEM operating under the pull-model. We modified the TIS/PEM implementation to support the pull-model in addition to the push-model. Instead of reading/writing the local certificate database, our modified TIS/PEM implementation issues requests to the centralized server to retrieve/store certificates. Before issuing requests, the TIS/PEM program sends a connection request to the server. This connection is tablished only once during a program execution, before any requests take place. If for some reason a TIS/PEM program is inactive for some time, or terminates prematurely before having a chance to disconnect itself, the server terminates the connection after waiting for a configurable time period. This prevents unnecessary tie-up of server resources.

The centralized server, as its name implies, manages certificates for multiple PEM clients. Our centralized server is able to accept requests from any PEM client operating in the Internet. However, it can be configured to provide services to a more restricted group of PEM clients based on their Internet addresses.

To achieve reasonable performance, the centralized server maintains certificates in a memory cache. Since cache space is limited, certificates are replaced as new ones are referenced. We investigated various cache replacement algorithms (FIFO, LRU, LFU) to find the optimal one. Furthermore, both write-through and write-back strategies were implemented to find the most efficient backing store management strategy. Hereafter, our server is called the cache server. 
In order to reduce the risk of denial-of-service attacks, the cache server performs certificate validation before it stores a certificate into the cache or to its backing store. This validation is independent of any validation that PEM clients perform for themselves. PEM clients must still execute their normal validation procedure since the communication between the cache server and its clients are via non-secure exchanges.

To ensure a smooth transition from the push-model to the pull-model, we implemented a PEM archive (a place in the designated DIT subtree writable by the cache server that we use as a backing store) to store certificates. The reason that we chose to store these certificates in the X.500 directory rather than in the local file system is that data in X.500 directory could be configured to be replicated, which adds redundancy. This meant we did not have to implement such replication ourselves. When the X.500 directory becomes more popular, the use of the PEM archive could be gradually eliminated. However, to work with existing PEM implementations, the cache server stores certificates only in its PEM archive, and retrieves certificates from their conventional places in the X.500 directory hierarchy only when they are not found in the PEM archive. To ensure only minimal changes are made to the TIS/PEM implementation, we modified the TIS/PEM routines that store/retrieve certificates to/from the local database so that they send certificate requests to the cache server instead. This guarantees that the user interface of TIS/PEM remains unchanged and the user should notice no difference in behavior other than changes in performance. We implemented and used in the cache server an X.500 library written with the ELROS programming system [Bran92].

Our choice of keeping certificates in cache server memory introduces other problems. In particular, if the cache server crashes, certificates that have not been written to the archive may be lost (this is only possible under a write-back strategy). 
However, we do not anticipate frequent server crashes, so we do not consider this to be significant. Furthermore, certificates are written to the archive within a very short period of time (i.e. a few minutes) after a storage request by a background activity. Loss of certificates due to a cache server crash is comparable to loss due to a TIS/PEM crash. In both cases, if the e-mail messages carrying the certificates can be reprocessed, the lost certificates can be recovered.

To support multiple clients concurrently, the server is multi-threaded. This way, multiple PEM clients can simultaneously send requests to the cache server and receive a timely reply, rather than experiencing long reply intervals due to the serialization of requests through a single threaded server. In addition, a multi-threaded server offers significant performance improvements for certificate storage operations when using a write-back strategy, since if requests are bursty, most "dirty" certificates can be written back by a background thread while the request activity is low.

In addition to caching certificates, our cache server also manages a cache of CRLs. While certificates and CRLs are managed in separate caches, they use identical cache management strategies. For example, if LRU is used as the replacement algorithm for the certificate cache, then it is also used as the replacement algorithm for the CRL cache. In our current implementation, we do not take into account of the problem of PEM clients not getting the most recent CRLs, since this is still a topic of on-going discussion in the PEM Working Group within the Internet Enginesring Task Force, a subsidiary group of the Internet Activities Board. Our implementation, however, can be modified easily to accommodate possible future enhancements.

The changes to the TIS/PEM implementation are limited to the low-level certificate/CRL retrieval/storage routines. These routines issue requests to the cache 
server instead of reading/writing the local database directly. The cache server handles four different types of retrieval/storage requests from the TIS/PEM clients. These requests are GET_CERT, PUT_CERT, GET_CRL, and PUT_CRL, which correspond to retrieving a certificate, storing certificates, retrieving a $\mathrm{CRL}$, and storing a $\mathrm{CRL}$, respectively.

Before placing a certificate or a CRL into the cache, the cache server performs a complete validation on it. Complete validation of a certificate involves checking its validity period, its signature, the subject and issuer's distinguished names (DNs) to see if they satisfy the subordination requirements specified in [RFC1422], and to ensure it has not been revoked. The only exception to a complete validation check is when a certificate or a CRL is retrieved from the archive. In this case only partial validation (no signature check, and in the case of a certificate, no check for DN subordination) is required, as complete validations are performed for all data stored in the archive.

When using write-back, the cache server defers writing replaced certificates or CRLs to the archive until after client requests are serviced. This allows it to provide a more responsive service. There is a background thread that processes certificates and CRLs that have been placed on a queue, either to be written to the archive or to be removed from it. The background thread does partial validation on certificates and CRLs before writing them to the archive or removing them from it. If the queue of requests is empty, the background thread sweeps the certificate and the CRL caches to see if there is any "dirty" certificate or CRL to be written.

For our tests we used the QUIPU implementation of X.500 both to hold the archive and to serve as the local DSA when searching for certificates and CRLs under the pull-model. 
Figure 6.0 depicts a scenario where the cache server is concurrently serving five PEM clients. The server creates a thread to service each PEM client. In addition, the server maintains a background thread for writing data to the archive under the writeback strategy. The server communicates with the X.500 DSA to read/write data to the PEM archive and to read data from the full X.500 hierarchy.

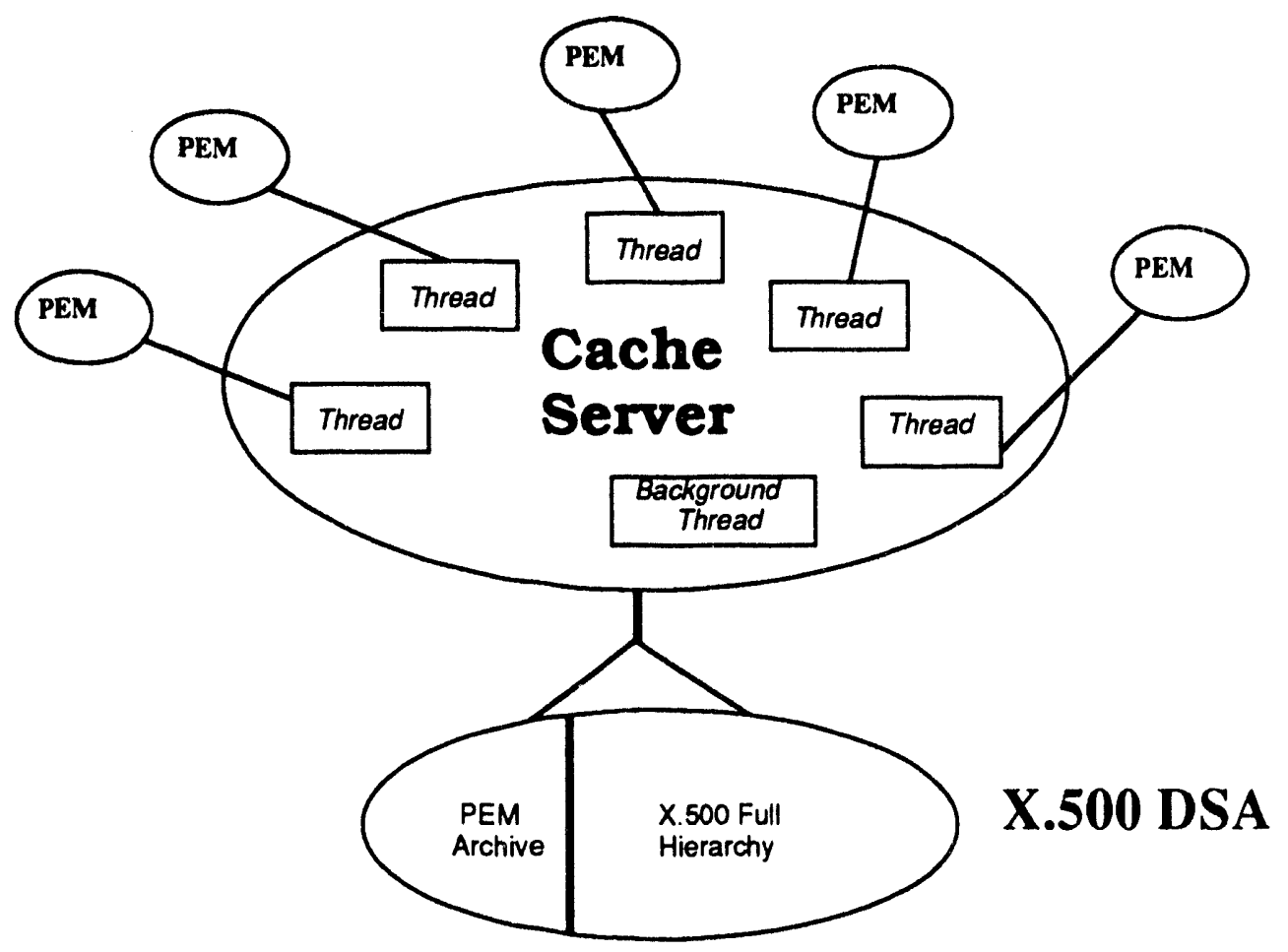

Figure 6.0 System Architecture 


\section{Cache replacement algorithms}

The number of certificates and CRLs used in our tests are quite limited (10 certificates and 5 CRLs). We would have used more if we could have obtained them, but since TIS/PEM is fairly new, not many certificates and CRLs were available. However, we believe the tests give us a good estimate about the performance of the cache server.

Each test processes privacy enhancements from 15 incoming messages and adds privacy enhancements to 10 outgoing messages. There are two types of incoming messages, one that includes the certification path of the originator, and the other that does not. Similarly, the outgoing messages can be sent with or without the certification path of the originator. In general, the messages with the certification path included are sent only once to a recipient in the very beginning of a sequence of exchanges, so that the recipient will have the necessary certificates in order to process the messages. The messages in each test generate a total of 142 GET_CERTs, 9 PUT_CERTs, 109 GET_CRLs, and 4 PUT_..CRLs.

We measured the performance of the cache server using three well-known cache replacement algorithms (FIFO, LRU, LFU). In addition, we developed a cache replacement algorithm that is customized to PEM certificate retrieval/storage patterns. 


\subsection{FIFO. LRU, and LFU}

FIFO is the simplest replacement algorithm. It always replaces the entry that has been held in the cache for the longest time. It is straightforward to implement, but since it does not take usage into account, FIFO tends to be perform poorly.

LRU is based on the locality principle, that is, that the more recently used entries will more likely be used again in the near future. Thus, LRU replaces the entry in the cache that has not been referenced for the longest time.

LFU is based on the idea that items used frequently in the recent past will more likely be used again in the near future. Thus, LFU replaces the entry in the cache that has been referenced the least number of times in the recent past.

\subsection{New replacement algorithm (DEPTH)}

The rationale for developing this new replacement algorithm is that while LRU and LFU take usage into account, they both have some drawbacks. LRU replaces a certificate no matter how often it is used, while in LFU, a certificate that was once referenced often may be kept in the cache for quite a long time, even though the certificate will not be referenced again in the near future. The new algorithm is designed to take advantage of certain reference locality properties associated with certification paths.

To design the algorithm so that it is customized to our application, we need to look at the pattern of cache server requests. We will use the messages in Figures 2.0 and 2.1 as examples. The two messages are sent by the same person. The DN of the 
person is $" / \mathrm{C}=\mathrm{US} / \mathrm{S}=\mathrm{MD} / \mathrm{O}=$ Trusted Information Systems $/ \mathrm{OU}=$ Glenwood/CN=James A. Galvin/." His CA's DN is "/C=US/S=MD/O=Trusted Information Systems/OU=Glenwood/," while the CA's CA (which is a PCA) has the DN "/C=US/S=MD/O=Trusted Information Systems PCA/."

TIS/PEM will process the two messages differently. For integrity and authentication checks, the processing will be the same. An unprivileged TIS/PEM program will issue a sequence of cache server requests to obtain the certificate for "/C=US/S=MD/O=Trusted Information Sysiems/OU=Glenwood/CN=James A. Galvin/," and to obtain other certificates and CRLs for validation of the user certificate. However, for the message in Figure 2.0, TIS/PEM will also start a privileged process to store the certificates on the certification path into the local database. The sequence of cache server requests issued by the privileged process is shown in Figure 7.0 (in this example, the certificates and CRLs for the CAs are already in the database, which should be true since in practice they are needed for the validation of the certificate of the user). The sequence of cache server requests issued by the unprivileged program to perform integrity and authentication checks is shown in Figure 7.1.

In both cases, there are many more GET requests than PUT requests for the processing of incoming messages. For generating outgoing messages, only GET requests are issued. For an outgoing message with certification path of the originator to be included in the message, there will be requests to retrieve all the certificates on the certification path, and corresponding requests to retrieve certificates and CRLs for validation, similar to that in Figure 7.0, except no PUT request is needed. For an outgoing message without certification path, there will be a request to retrieve the certificate of the originator and corresponding requests to retrieve certificates and CRLs for the validation of that user certificate, similar to that in Figure 7.1. 
GET_CERT for "C=US/S=MD/O=Trusted Information Systems /OU=Glenwood/CN=James A. Galvin/"

GET_CERT for "C=US/S=MD/O=Trusted Information Systems /OU=Glenwood/"

GET_CRL for "C=US/S=MD/O=Trusted Information Systems /OU=Glenwood/"

GET_CERT for "C=US/S=MD/O=Trusted Information Systems PCA"

GET_CRL for "C=US/S=MD/O=Trusted Information Systems PCA"

GET_CERT for "C=US/S=MD/O=Trusted Information Systems PCA"

GET_CRL for "C=US/S=MD/O=Trusted Information Systems PCA"

PUT_CERT for "C=US/S=MD/O=Trusted Informations Systems /OU=Glenwood/CN=James A. Galvin/"

GET_CERT for "C=US/S=MD/O=Trusted Information Systems /OU=Glenwood/"

GET_CERT for "C=US/S=MD/O=Trusted Information Systems PCA"

GET_CRL for "C=US/S=MD/O=Trusted Information Systems PCA"

GET_CERT for "C=US/S=MD/O=Trusted Information Systems PCA"

GET_CRL for "C=US/S=MD/O=Trusted Information Systems PCA"

GET_CERT for "C=US/S=MD/O=Trusted Information Systems PCA"

GET_CERT for "C=US/S=MD/O=Trusted Information Systems PCA"

GET_CRL for "C=US/S=MD/O=Trusted Information Systems PCA"

Figure 7.0 Sequence of cache server requests issued by a privileged process in processing an incoming message with certification path included 
GET_CERT for "C=US/S=MD/O=Trusted Information Systems /OU=Glenwood/"

GET_CRL for "C=US/S=MD/O=Trusted Information Systems /OU=Glenwood/"

GET_CERT for "C=US/S=MD/O=Trusted Information Systems PCA"

GET_CRL for "C=US/S=MD/O=Trusted Information Systems PCA"

GET_CERT for "C=US/S=MD/O=Trusted Information Systems PCA"

GET_CRL for "C=US/S=MD/O=Trusted Information Systems PCA"

Figre 7.1 Sequence of cache server requests issued by the unprivileged program during the processing of an incoming message to perform integrity and authentication checks

Looking at the GET_CERT requests in Figure 7.0, we notice that there are more requests for " $\mathrm{C}=\mathrm{US} / \mathrm{S}=\mathrm{MD} / \mathrm{O}=$ Trusted Information Systems $\mathrm{PCA}$ " than any others. This is because for each certificate on the certification path, in order to validate it, its issuer's certificate is needed for validation, and the issuer's issuer's certificate is needed for the validation of the issuer's certificate, and this process continues on all the way to the PCA certificate. In Figure 7.0, the first 4 GET_CERTs are used for validation of the certificate for $\quad \mathrm{C}=U \mathrm{U} / \mathrm{S}=\mathrm{MD} / \mathrm{O}=\mathrm{Trusted}$ Information Systems/OU=Glenwood/CN=James A. Galvin/," while the next 3 GET_CERTs are used for validation of the certificate for $" \mathrm{C}=\mathrm{US} / \mathrm{S}=\mathrm{MD} / \mathrm{O}=$ Trusted Informations Systems/OU=Glenwood/," and the last 2 GET_CERTs are used for the validation of the certificate for "C=US/S=MD/O=Trusted Information Systems PCA." The reason that TIS/PEM clients issue so many requests is that TIS/PEM is designed to access a local database, and TIS/PEM has a mechanism of marking a certificate (or CRL) as valid once validation is performed on it, so the next time it is accessed no validation is required. However, in our implementation, communication between the TIS/PEM clients and the 
cache server is not secure. Hence, every certificate obtained from the cache server must be validated.

We also note that while there is only one GET_CERT for "C=US/S=MD/O=Trusted Information Systems/OU=Glenwood/ $\mathrm{CN}=\mathrm{James} A$. Galvin/," there are two GET_CERTs for "C=US/S=MD/O=Trusted Informations Systems/OU=Glenwood/," and 6 GET_CERTs for "C=US/S=MD/O=Trusted Information Systems PCA." This shows that many requests are redundant, an undesired result of the original TIS/PEM design that assumes certificates need to be validated only once. This is a suboptimal design. The optimal design should eliminate duplicate requests by remembering what had already been validated and processing certificates and CRLs in a more appropriate order. Thus, the ideal sequence of requests for the same incoming message is shown in Figure 7.2.

GET_CERT for "C=US/S=MD/O=Trusted Information Systems /OU=Glenwood/CN=James A. Galvin/"

GET_CERT for "C=US/S=MD/O=Trusted Information Systems /OU=Glenwood/"

GET_CRL for "C=US/S=MD/O=Trusted Information Systems /OU=Glenwood/"

GET_CERT for "C=US/S=MD/O=Trusted Information Systems PCA"

GET_CRL for "C=US/S=MD/C=Trusted Information Systems PCA"

PUT_CERT for "C=US/S=MD/O=Trusted Information Systems /OU=Glenwood/CN=James A. Galvin/"

Figure 7.2 Ideal sequence of cache server requests during the processing of an incoming message with certification path included

To realize the optimal design we have in mind would require major modifications to TIS/PEM, which is out of the scope of this research. However, we 
designed our cache replacement algorithm based on the optimal cache server request pattern.

Suppose now we have another user named "UserA" who is an employee at TIS at Glenwood. His/her DN would very likely be "C=US/S=MD/O=Trusted Information Systems/OU=Glenwood/CN=UserA/." The ideal sequence of requests resulting from processing an incoming message from UserA is shown in Figure 7.3.

GET_CERT for "C=US/S=MD/O=Trusted Information Systems /OU=Glenwood/CN=UserA/"

GET_CERT for "C=US/S=MD/O=Trusted Information Systems /OU=Glenwood/"

GET_CRL for "C=US/S=MD/O=Trusted Information Systems /OU=Glenwood/"

GET_CERT for "C=US/S=MD/O=Trusted Information Systems PCA"

GET_CRL for "C=US/S=MD/O=Trusted Information Systems PCA"

PUT_CERT for "C=US/S=MD/O=Trusted Information Systems /OU=Glenwood/CN=UserA/"

Figure 7.3 Ideal sequence of cache server requests during the processing of an incoming message with certification path included

Next, if we have a user whose name is "UserB" as an employee of TIS at Mountain View, his/her DN would very likely be $" \mathrm{C}=\mathrm{US} / \mathrm{S}=\mathrm{MD} / \mathrm{O}=$ Trusted Information Systems/OU=Mountain View/CN=UserB/." The ideal sequence of requests resulting from processing an incoming message from UserB is shown in Figure 7.4. 
GET_CERT for "C=US/S=MD/O=Trusted Information Systems /OU =Mountain View/CN=UserB $/$ "

GET_CERT for "C=US/S=MD/O=Trusted Information Systems /OU $=$ Mountain View/"

GET_CRL for "C=US/S=MD/O=Trusted Information Systems /OU $=$ Mountain View/"

GET_CERT for "C=US/S=MD/O=Trusted Information Systems PCA"

GET_CRL for "C=US/S=MD/O=Trusted Information Systems PCA"

PUT_CERT for "C=US/S=MD/O=Trusted Information Systems /OU =Mountain View/CN=UserB/"

Figure 7.4 Ideal sequence of cache server requests during the processing of an incoming message with certification path included

In Figure 7.1, we see that the sequence of cache server requests issued by an unprivileged process for integrity and authenticity checks is already close to optimal. The only exception is that there are 2 GET_CERT requests for the PCA. This is due to the fact that "C=US/S=MD/O=Trusted Information Systems PCA" is currently acting as the IPRA, since the IPRA is not yet operational in the Internet. Once the IPRA is functional, there will only be one GET_CERT request for the PCA.

Careful examination of Figures 7.1, 7.2, 7.3, and 7.4 indicates that certificates and CRLs for entities higher up in the certification hierarchy are referenced more often than those at the lower end of the hierarchy, since users share a common subhierarchy at the higher end. This observation prompts us to use the depth of a certificate or CRL at the certification hierarchy as an input in deciding which certificates or CRLs to replace. This favors certificates and CRLs of entities at the higher end of the certification hierarchy. 
Another observation is that we do not want to keep certificates in the cache if they are not referenced in the recent past, even if they are at the higher end of the certification hierarchy. We do not want those certificates to stay in the cache for too long. Thus, our algorithm should not only take into consideration the depth of a certificate or CRL but also when it was last referenced. In the following discussions, we will discuss our design in terms of certificates only, since the CRL cache has an identical design.

With the above considerations in mind, I designed the following cache replacement algorithm, named DEPTH. DEPTH has two parameters. The first parameter is the depth of a certificate, that is, the depth of the certificate in its Internet certification hierarchy. Thus, from Figure 2.4, the depth of the certificate for TIS PCA is 1 , while that for LLNL is 2 , and so on. The other parameter is the relative order of the most recent reference of a certificate. Thus, the most recently referenced certificate has a value of 1 , the next most recently referenced certificate has a value of 2 , and so on, while the least recently used certificate has a value equal to the number of certificates in the cache.

The DEPTH algorithm is designed based on priority queuing concepts. The number of queues is equal to the maximum depth in the certification hierarchy. The queues are numbered sequentially from 1 to the maximum number of queues. A new certificate brought into the cache is placed initially at the end of a queue whose queue number is equal to that of the depth of the certificate in its certification hierarchy. During replacement, we replace the certificate at the beginning of the highest numbered non-empty queue. 
It is evident that a design based simply on the priority queuing scheme will result in some certificates being held in the cache forever despite their usage. This situation will arise if there is always some certificate at the lower part of the certification hierarchy in the queue even though those certificates at the higher part of the hierarchy will never be used again. What we need is a mechanism to age certificates and demote them if they have not been used for a certain period of time. Thus, we come up with the following supplemental aging algorithm.

When the certificate being replaced is not the least recently used one, we find the least recently used certificate, and demote it to the next higher numbered (lower priority) queue, and make it the most referenced one. This way, no certificates will be held in the queue forever. Every certificate will eventually be replaced after some number of replacements if it has not been referenced in the duration. If a certificate is referenced, not only is it marked as being the most recently referenced certificate, but also it is removed from the current queue and placed at the end of the queue whose number equals the depth of the certificate. We find that the maximum number of replacements that it takes to replace a certificate if it is not referenced again is described precisely by the following formula. Let $S$ be the size of the cache (maximum number of cache entries), $\mathrm{D}$ be the maximum depth of the certification hierarchy, and $\mathrm{d}$ be the depth of a certificate in the hierarchy, then the maximum number of replacements it takes to replace the certificate if it is not referenced again is:

$$
S *(D-d+1)
$$

It is evident that the larger the value of $d$, which means the certificate is at the lower end of the certification hierarchy, the smaller is maximum number of replacements it takes to replace it. This is consistent with the intent of our design. 


\section{Performance Results}

To analyze the performance of the cache server, two types of measurements are used. One is the response time of requests to the cache server and the other is the cache hit ratios that result from the requests. We define response time as the time interval between the sending of a retrieval/storage request and the receiving of the corresponding result from the cache server. In measuring response time, we have chosen to ignore the time it takes to make a connection to the cache server since the connection occurs only once in the execution of the TIS/PEM program. Cache hit ratio is defined as the total number of cache hits divided by the total number of cache accesses.

Response times can vary greatly depending on the load in the machine where the tests run. We ran our tests at night and weekends so that the effect of load activity on real time measurements is minimized.

We decided to measure the response times and the hit ratios in separate tests because writing the hit ratio results to files would give inaccurate response times. However, the response times for tests using the write-back strategy could vary slightly from one test to another, even though those under the write-through strategy are always consistent. The reason for the variation under the write-back strategy is that some data could be fetched from the queue serviced by the background thread, 1 which could be time dependent. When data is found in the queue, we treated that as a hit, since there is no X.500 operation involved. The variations are very small, so the hit ratios are still good enough to serve as a reference.

1 In our implementation, when using the write-back strategy, if data is not found in a cache, the queue serviced by the background thread is searched. If data is found in the queue, then X.500 operations are not necessary. This feature is designed to solve the problem when GET requests try to obtain data that is still waiting to be written to the X.500 directory by the background thread. It turns out that occasionally this can save a few reads to the X.500 directory. 
Figure 8.0 shows the configuration for the tests. The cache server, QUIPU, and PEM clients run on three different machines connected via Ethernet. The cache server runs on a diskless SPARCstation SLC with 16 megabytes of memory, while QUIPU runs on a SPARCstation 2 with 64 megabytes of memory, and the PEM clients run on a SUN 4/690 with 128 megabytes of memory.

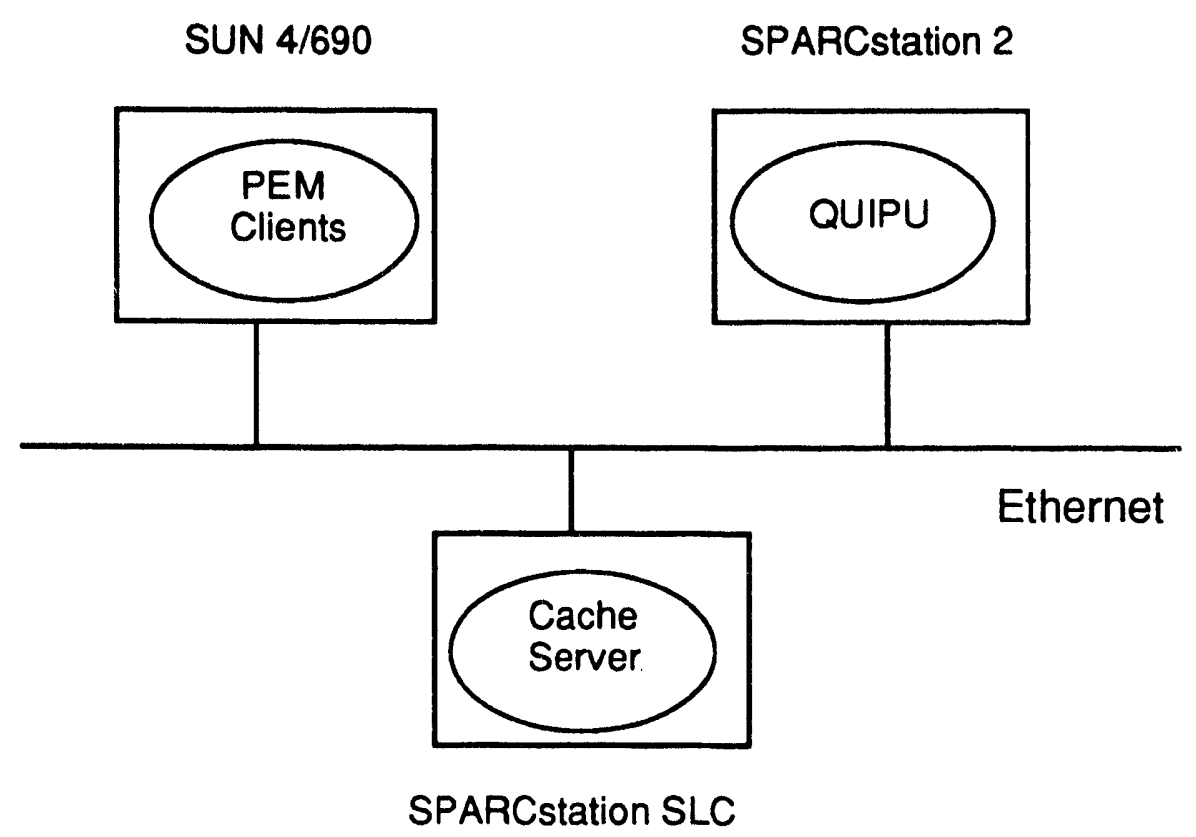

Figure 8.0 Configuration

In the following tables, the parameter used for the certificate cache size is the number of certificates in the certificate cache, and the parameter for the CRL cache size is the number of CRLs in the CRL cache. The response times are measured in seconds. For comparison purposes, we have also included the local file access times for the original PEM file accesses (obtained on the SUN 4/690 and indicated by "Local" in the tables), the access times when files are accessed via NFS (also obtained on the SUN 
$4 / 690$, and indicated by "NFS" in the tables), and the response times when no cache is used in the cache server (indicated by "No Cache" in the tables).

We ran tests with certificate caches of size $1,3,7$, and 10, and CRL caches of size 1 and 5. We only display one test result for certificate cache sizes of 1 and 10 , since our preliminary results show that there is no significant difference between response times obtained under different cache replacement algorithms for these certificate cache sizes, and thus there is no point in displaying them more than once (the results displayed are for FIFO). The response times are about the same since replacement algorithms do not come into play for these certificate cache sizes. When the cache size is 1 , there is only one certificate to replace, and when the cache size is 10 , there is no need to replace any certificate.

The response times shown in the tables for the case of no cache are probably slightly higher than what they really should be. The reason is that for our tests all requests go through the cache server, while in practice, if no caching was employed, requests would go directly to an X.500 directory server. But the communication times between the cache server and the PEM clients are very small compare to the overall times, so the response times for the case of no cache is still representative for analyzing performance with or without the use of cache. 


\begin{tabular}{|c|c|c|c|c|c|c|c|c|c|}
\hline & & \multicolumn{2}{|c|}{ GET_CERT } & \multicolumn{2}{|c|}{ PUT_CERT } & \multicolumn{2}{|c|}{ GET_CRL } & \multicolumn{2}{|c|}{ PUT_CRL } \\
\hline Local & & \multicolumn{2}{|c|}{.020} & \multicolumn{2}{|c|}{.290} & \multicolumn{2}{|c|}{.018} & \multicolumn{2}{|c|}{.158} \\
\hline NFS & & \multicolumn{2}{|c|}{.026} & \multicolumn{2}{|c|}{1.584} & \multicolumn{2}{|c|}{.024} & \multicolumn{2}{|c|}{.784} \\
\hline \multirow[t]{3}{*}{ No Cache } & & 2.0 & & 2.8 & & & & 2.6 & \\
\hline & $\begin{array}{l}\text { CERT } \\
\text { CACHE } \\
\text { SIZE }\end{array}$ & \multicolumn{8}{|c|}{$\begin{array}{c}\text { CRL } \\
\text { CACHE } \\
\text { SIZE }\end{array}$} \\
\hline & & 1 & 5 & & 5 & & 5 & 1 & 5 \\
\hline & 1 & 1.380 & 1.202 & 4.525 & 3.950 & .585 & .134 & 4.200 & 4.233 \\
\hline \multirow[t]{2}{*}{ FIFO } & 3 & .928 & .716 & 3.749 & 3.150 & .589 & .133 & 2.777 & 2.911 \\
\hline & 7 & .582 & 347 & 3.728 & 3.108 & .582 & .135 & 2.801 & 2.865 \\
\hline \multirow[t]{2}{*}{$\overline{\text { LRU }}$} & $\overline{3}$ & .941 & 736 & 3.800 & 3.602 & .588 & .136 & 2.764 & 2.796 \\
\hline & 7 & .538 & .286 & 3.760 & 3.026 & .588 & .129 & 2.792 & 2.765 \\
\hline \multirow[t]{2}{*}{$\overline{\mathrm{LFU}}$} & 3 & .843 & .661 & 3.824 & 3.131 & .585 & .129 & 2.750 & 2.810 \\
\hline & 7 & .533 & .290 & 3.695 & 3.139 & .581 & .130 & 2.723 & 2.734 \\
\hline \multirow[t]{3}{*}{ DEPTH } & 3 & 831 & .658 & 3.805 & 3.017 & .587 & .127 & 2.757 & 2.754 \\
\hline & 7 & $\overline{531}$ & .287 & 3.711 & 3.016 & .580 & .131 & 2.729 & 2.721 \\
\hline & 10 & .534 & .286 & 3.723 & 3.012 & .585 & .126 & 2.795 & 2.783 \\
\hline
\end{tabular}

Table 8.0 Response times for tests using write-through with validations (in secs)

\begin{tabular}{|c|c|c|c|c|c|c|c|c|c|}
\hline & & \multicolumn{2}{|c|}{ GET_CERT } & \multicolumn{2}{|c|}{ PUT_CERT } & \multicolumn{2}{|c|}{ GET_CRL } & \multicolumn{2}{|c|}{ PUT_CRL } \\
\hline Local & & \multicolumn{2}{|c|}{.020} & \multicolumn{2}{|c|}{.290} & \multicolumn{2}{|c|}{.018} & \multicolumn{2}{|c|}{.158} \\
\hline NFS & & \multicolumn{2}{|c|}{.026} & \multicolumn{2}{|c|}{1.584} & \multicolumn{2}{|c|}{.024} & \multicolumn{2}{|c|}{.784} \\
\hline $\begin{array}{c}\text { No } \\
\text { Cache }\end{array}$ & & \multicolumn{2}{|c|}{2.027} & \multicolumn{2}{|c|}{2.881} & \multicolumn{2}{|c|}{1.778} & \multicolumn{2}{|c|}{2.680} \\
\hline & $\begin{array}{c}\text { CERT } \\
\text { CACHE } \\
\text { SIZE }\end{array}$ & \multicolumn{8}{|c|}{$\begin{array}{c}\text { CRL } \\
\text { CACHE } \\
\text { SIZE }\end{array}$} \\
\hline & & 1 & 5 & 1 & 5 & 1 & 5 & 1 & 5 \\
\hline & $T$ & 1.443 & 1.127 & 2.017 & 1.353 & .678 & .199 & 1.645 & 1.670 \\
\hline \multirow[t]{2}{*}{ FIFO } & 3 & .952 & .688 & 1.182 & .512 & .675 & .179 & .092 & .089 \\
\hline & 7 & .562 & .288 & 1.000 & .475 & .612 & .178 & .088 & .086 \\
\hline \multirow[t]{2}{*}{$\overline{\mathrm{LRU}}$} & $\overline{3}$ & .966 & .698 & 1.000 & .486 & .577 & .145 & .087 & .092 \\
\hline & 7 & .536 & .262 & 1.169 & .478 & .663 & .195 & .087 & .090 \\
\hline \multirow[t]{2}{*}{ LFU } & 3 & .807 & .606 & 1.044 & .504 & .662 & .158 & .092 & .094 \\
\hline & 7 & .555 & .282 & 1.164 & .518 & .679 & .157 & .087 & .107 \\
\hline \multirow[t]{3}{*}{$\overline{\text { DEPTH }}$} & 3 & .802 & .596 & 1.025 & .495 & .653 & .151 & .085 & .091 \\
\hline & 7 & .538 & .257 & 1.094 & .483 & .661 & .143 & .083 & .082 \\
\hline & 10 & .523 & .247 & .999 & .520 & .598 & .173 & .087 & .119 \\
\hline
\end{tabular}

Table 8.1 Response times for tests using write-back with validations (in secs)

From Table 8.0, it is evident that when using the write-through strategy, the GET_CERT and GET_CRL requests are more efficient with caches of any size than that 
without the use of cache. The PUT_CERT and PUT_CRL requests perform slightly worse, but they are not as frequent in PEM applications (less than $10 \%$ of requests resulting from processing PEM messages in our tests are PUT requests). Moreover, from Table 8.1 where the response times when using the write-back scheme are recorded, both the GET and PUT requests perform better with the use of caches. Thus, certificate and CRL caches improve the performance of a central certificate server.

In theory the response times for GET requests using the write-back strategy should be larger than those for the write-through strategy, since writes performed by the background thread can interfere with the GET requests. However, due to the fact that some data could be found in the queue serviced by the background thread, the GET requests for the write-back strategy can sometimes perform better. Hence, the response times for GET requests are quite close under either strategy. With the significant improvement in the response times for the PUT requests, it is safe to conclude that the write-back strategy is a better choice than the write-through strategy.

As can be seen, increasing certificate cache size produces bigger performance gain when the cache sizes are smaller. Increasing the certificate cache size from 1 to 3 reduces $30-40 \%$ of the response times for GET_CERT. Response times when the certificate cache size is 7 shows that they are about the same as those for certificate cache size of 10 , which suggests that increasing cache sizes beyond approximately $70 \%$ of the total number of certificates stored wastes the extra memory resources required.

Increasing CRL cache size not only improves performance for GET_CRL, but also improves performance for the certificate requests. This effect is a result of validations. Partial validations are performed when retrieving a certificate, and complete 
validations are performed when storing a certificate. Validation of a certificate requires its issuer's CRL.

To analyze the performance of the cache replacement algorithms, we have to understand how the certificates are accessed. In processing a message, all the certificates on the certification path of the sender must be brought into the cache, with the certificates higher up the path accessed more often than those below. In our tests, the number of certificates on the certification path of any user is either 2 or 3 . The users with the same length of certification path happen to have the same PCA, while those with different lengths have a different PCA.

The response times for the different replacement algorithms do not seem to yield any significant difference, due to the following reasons. We will first analyze the case when the certificate cache size is 3 . In this case, either most or all of the certificates in the cache are replaced when a new message is being processed, if consecutive messages are sent by users with different certification paths, which is the case in our tests. The above scenario is true no matter what replacement algorithm is used. LFU and DEPTH seem to perform slightly better in this case, because the PCA certificate for the sender with certification path of length 3 , which is accessed the most often, can survive over message boundaries. The PCA certificate for the sender with certification path of length 2 cannot survive because when the next message processed is sent by a user with certification path of length 3 , the certificates from the new message will fill up the whole cache.

For the case when the certificate cache size is 7, LRU, LFU, and DEPTH seem to achieve nearly the maximum performance possible, while FIFO is very close. The reason is that 7 is more than twice as large as 3 , which means the cache is large enough 
to hold all the certificates for the senders of any two messages. Also, 7 is very close to 10 , the total number of certificates. A few certificates are accessed at one point and never again. Hence, any reasonable algorithm should be able to keep the certificates likely to be accessed again in the cache. FIFO performs slightly worse since it does not take usage into account at all, and can throw away PCA certificates, while LRU, LFU, and DEPTH are not likely to do so, since PCA certificates are accessed the most often, and thus usually fairly recently.

However, the response times do not vary significantly enough for us to conclude that one algorithm is indeed better than the others, although it is quite evident that FIFO is not likely to be any better than the other three under any circumstances. Tests with a larger number of certificates and CRLs might help discriminate between LRU, LFU, and DEPTH.

Figure 8.1 provides another illustration of the relative performance of the various algorithms. It shows that LFU and DEPTH have slightly better performance than FIFO and LRU under all circumstances. Since both LFU and DEPTH replaces certificates based on the frequency of access to certificates, this is an indication that frequency of access is a better usage factor in the replacement decision. On the other hand, LFU and DEPTH perform similarly because they both use the frequency of access to determine what to replace next, even though the parameters they use are different - LFU uses a frequency count and DEPTH uses the depth of a certificate. Both parameters end up giving similar preference to certificates. 


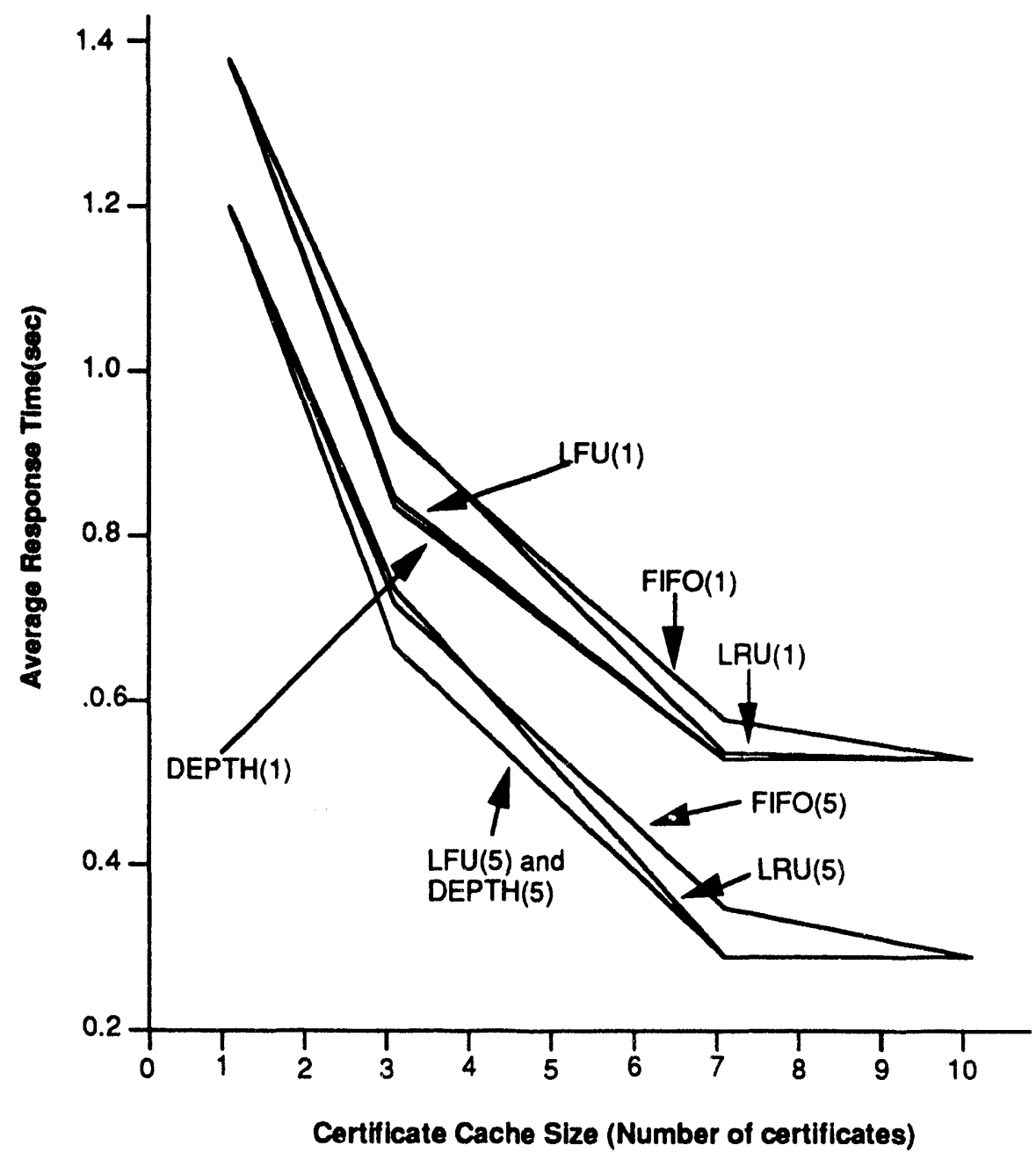

Figure 8.1 Comparison of average response time in relation to cache size for GET_CERT (the number in parentheses is the CRL cache size) using write-through

Tables 8.0 and 8.1 show that the response times for GET requests using the cache server are worse than the file access times (both local and NFS). Even the lowest response times for GET_CERT requests are still more than 12 times that of corresponding local file access times and more than 9 times the file access times when NFS is used. Similarly, the lowest response times for GET_CRL requests are still about 7 times that of corresponding local file access times and more than 5 times the file access times when NFS is used. When a write-back strategy is used, since the cache server responds immediately without waiting for the actual writes to take place, the 
response times for PUT_CRL requests can be up to twice as fast as the local file access times and 9 times as fast as the file access via NFS. The response times for the PUT_CERT requests under a write-back strategy can be twice as long as local file access times while 3 times as fast as file access via NFS. Since PUT requests are more rare, overall, the cache server requests will take longer than either the local file accesses or remote file accesses. This is the type of trade-off associated with distributed access versus local access and higher level of security versus minimum security. We believe the increased availability of certificates is worth the extra delay. Furthermore, we have not performed any optimization on the cache server yet, so the extra delay may not be as great as what we see here. Finally, it is hard to measure how the increased availability of certificates stored in a central certificate server will improve performance on average. This is a topic for future study.

Another way to analyze the performance is to observe each individual response time. We notice that the majority of GET requests actually perform quite well, except for a few cases when the cache server goes to the directory for data retrieval. These few cases result in response times that are much higher than those when the data needed can be satisfied by the cache alone, thus bringing the average response times up significantly. For example, in Table 8.1, the average response time for GET_CRL when using LFU with a certificate cache of size 7 and a CRL cache of size 5 is .157 seconds. However, out of the 109 response times, 6 of them have an average of 2.229 seconds, while the remaining 103 average .037 seconds, much lower than the average of .157 seconds. The median for the 109 response times is .032 seconds. This observation suggests that most cache server retrieval requests perform fairly well, while a small portion of requests will experience significant performance delay. We use this fact in Section 10 to suggest a way to significantly improve cache server performance. 


\begin{tabular}{|c|c|c|c|c|c|}
\hline & & \multicolumn{3}{|c|}{ GET_CERT } & \multicolumn{2}{c|}{ GET_CRI } \\
\hline \multirow{4}{*}{$\begin{array}{c}\text { CERT } \\
\text { CACHE } \\
\text { SIZE }\end{array}$} & \multicolumn{4}{|c|}{$\begin{array}{c}\text { CACHE } \\
\text { SIZE }\end{array}$} \\
\hline & & 1 & 5 & 1 & 5 \\
\hline & 1 & .446 & .446 & .582 & .955 \\
\hline FIFO & 3 & .712 & .716 & .582 & .955 \\
\cline { 2 - 6 } & 7 & .886 & .886 & .582 & .955 \\
\hline LRU & 3 & .710 & .710 & .582 & .955 \\
\cline { 2 - 6 } & 7 & .911 & .911 & .582 & .955 \\
\hline LFU & 3 & .761 & .761 & .582 & .955 \\
\cline { 2 - 6 } & 7 & .911 & .911 & .582 & .955 \\
\hline DEPTH & 3 & .761 & .761 & .582 & .955 \\
\cline { 2 - 6 } & 7 & .911 & .911 & .582 & .955 \\
\hline & 10 & .911 & .911 & .582 & .955 \\
\hline
\end{tabular}

Table 8.2 Hit ratios for tests using write-thru with validations ( 0 for PUT_CERT and PUT_CRL requests)

\begin{tabular}{|c|c|c|c|c|c|}
\hline & & \multicolumn{2}{|c|}{ GET_CERT } & \multicolumn{2}{c|}{ GET_CRL } \\
\hline \multirow{4}{*}{$\begin{array}{c}\text { CERT } \\
\text { CACHE } \\
\text { SIZE }\end{array}$} & \multicolumn{4}{|c|}{$\begin{array}{c}\text { CRL } \\
\text { SIZEE }\end{array}$} \\
\hline & & 1 & 5 & 1 & 5 \\
\hline & 1 & .465 & .477 & .579 & .955 \\
\hline FIFO & 3 & .716 & .716 & .582 & .955 \\
\cline { 2 - 6 } & 7 & .886 & .886 & .582 & .955 \\
\hline LRU & 3 & .710 & .710 & .582 & .955 \\
\cline { 2 - 6 } & 7 & .911 & .911 & .607 & .955 \\
\hline LFU & 3 & .773 & .773 & .582 & .955 \\
\cline { 2 - 6 } & 7 & .911 & .911 & .582 & .955 \\
\hline DEPTH & 3 & .761 & .761 & .607 & .955 \\
\cline { 2 - 6 } & 7 & .911 & .911 & .582 & .955 \\
\hline & 10 & .911 & .911 & .607 & .955 \\
\hline
\end{tabular}

Table 8.3 Hit ratios for tests using write-back with validations ( 0 for PUT_CERT and PUT_CRL requests)

We also present the cache hit ratios of the tests in Tables 8.2 and 8.3. Cache hit ratios for PUT_CERT and PUT_CRL are not shown because they are always 0 . The reason is that TIS/PEM does not store a certificate if it is already in the database, and to be consistent with certificate requests, we choose to store CRLs only once also, which is 
reasonable since in practice the issuance of CRLs will generally be much less often than their accesses.

The cache hit ratios are quite consistent with the response times obtained. The tests with lower response times show higher hit ratios, and those with close response times have similar hit ratios.

\subsection{No Validation}

Since validations are performed in response to a potential security threat, we wanted to determine the cost associated with this security measure. Tables 8.4 through 8.7 show the cache server performance when operating without validation. There is no measurements for the DEPTH algorithm in these tables because the depth of a certificate in a certification path is calculated during validation, so validation is part of the DEPTH algorithm. Figures 8.2 and 8.3 compare the performance of LFU with or without validation.

\begin{tabular}{|c|c|c|c|c|c|c|c|c|c|}
\hline & & \multicolumn{2}{|c|}{ GET_CERT } & \multicolumn{2}{|c|}{ PUT_CERT } & \multicolumn{2}{|c|}{ GET_CRL } & \multicolumn{2}{|c|}{ PUT_CRI } \\
\hline Local & & \multicolumn{2}{|c|}{.020} & \multicolumn{2}{|c|}{.290} & \multicolumn{2}{|c|}{.018} & \multicolumn{2}{|c|}{.158} \\
\hline NFS & & \multicolumn{2}{|c|}{.026} & \multicolumn{2}{|c|}{1.584} & \multicolumn{2}{|c|}{.024} & \multicolumn{2}{|c|}{.784} \\
\hline $\begin{array}{c}\text { No } \\
\text { Cache }\end{array}$ & & \multicolumn{2}{|c|}{2.027} & \multicolumn{2}{|c|}{2.881} & \multicolumn{2}{|c|}{1.778} & \multicolumn{2}{|c|}{2.680} \\
\hline & \begin{tabular}{|l} 
CERT \\
CACHE \\
SIZE
\end{tabular} & \multicolumn{8}{|c|}{$\begin{array}{c}\text { CRL } \\
\text { CACHE } \\
\text { SIZE }\end{array}$} \\
\hline & & & 5 & 1 & 5 & 1 & 5 & 1 & 5 \\
\hline & 1 & 1.096 & 1.093 & 2.983 & 2.966 & .726 & .167 & 2.716 & 2.718 \\
\hline \multirow[t]{2}{*}{ FIFO } & $\overline{3}$ & .662 & .673 & 2.897 & 2.929 & .720 & .169 & 2.754 & 2.736 \\
\hline & 7 & .292 & .273 & 2.942 & 2.900 & .735 & .165 & 2.734 & 2.730 \\
\hline \multirow[t]{2}{*}{$\overline{\mathrm{LRU}}$} & 3 & .649 & .648 & 2.906 & 2.891 & .719 & .165 & 2.696 & 2.752 \\
\hline & 7 & .223 & .223 & 2.966 & 2.948 & .732 & .169 & 2.700 & 2.706 \\
\hline \multirow[t]{3}{*}{ LFU } & $\overline{3}$ & .538 & .549 & 2.906 & 2.932 & .720 & .169 & 2.696 & 2.704 \\
\hline & $\overline{7}$ & .220 & .225 & 2.905 & 2.915 & .719 & .164 & 2.683 & 2.695 \\
\hline & 10 & .222 & .220 & 2.970 & 2.932 & .714 & .165 & 2.698 & 2.703 \\
\hline
\end{tabular}

Table 8.4 Response times for tests using write-through without validations (in secs) 


\begin{tabular}{|c|c|c|c|c|c|c|c|c|c|}
\hline & & \multicolumn{2}{|c|}{ GET_CERT } & \multicolumn{2}{|c|}{ PUT_CERT } & \multicolumn{2}{|c|}{ GET_CRL } & \multicolumn{2}{|c|}{ PUT_CRL } \\
\hline Local & & \multicolumn{2}{|c|}{.020} & \multicolumn{2}{|c|}{.290} & \multirow{2}{*}{\multicolumn{2}{|c|}{$\frac{.018}{024}$}} & \multirow{2}{*}{\multicolumn{2}{|c|}{$\frac{.158}{784}$}} \\
\hline NFS & & \multicolumn{2}{|c|}{.026} & \multicolumn{2}{|c|}{1.584} & & & & \\
\hline $\begin{array}{l}\text { No } \\
\text { Cache } \\
\text { cols }\end{array}$ & & \multicolumn{2}{|c|}{$\frac{.020}{2.027}$} & \multicolumn{2}{|c|}{$\frac{1.507}{2.881}$} & \multicolumn{2}{|c|}{1.778} & \multicolumn{2}{|c|}{2.680} \\
\hline & $\begin{array}{l}\text { CERT } \\
\text { CACHE } \\
\text { SIZE }\end{array}$ & \multicolumn{8}{|c|}{$\begin{array}{c}\text { CRL } \\
\text { CACHE } \\
\text { SIZE }\end{array}$} \\
\hline & & 1 & 5 & 1 & 5 & 1 & 5 & 1 & 5 \\
\hline & $T$ & 1.081 & 1.068 & .025 & .038 & .796 & .235 & .020 & .041 \\
\hline \multirow{2}{*}{ FIFO } & $\overline{3}$ & .729 & .720 & .028 & .032 & .748 & .179 & .021 & .026 \\
\hline & 7 & .245 & .293 & .024 & .027 & .728 & .200 & .020 & .019 \\
\hline \multirow{2}{*}{$\overline{\text { LRU }}$} & $\overline{3}$ & .714 & .682 & .043 & .024 & .762 & .218 & .025 & .020 \\
\hline & 7 & .234 & .222 & .026 & .025 & .806 & .202 & .020 & .019 \\
\hline \multirow[t]{3}{*}{ LFU } & 3 & .608 & .634 & .025 & .029 & .825 & .247 & .020 & .022 \\
\hline & 7 & .233 & .258 & .033 & .027 & .815 & .234 & .026 & .022 \\
\hline & 10 & .219 & .238 & .025 & .024 & .782 & .177 & .020 & .022 \\
\hline
\end{tabular}

Table 8.5 Response times for tests using write-back without validations (in secs)

\begin{tabular}{|c|c|c|c|c|c|}
\hline & & \multicolumn{3}{|c|}{ GET_CERT } & \multicolumn{2}{c|}{ GET_CRL } \\
\hline \multirow{4}{*}{$\begin{array}{c}\text { CRL } \\
\text { CACHE } \\
\text { SIZE } \\
\text { CACHE } \\
\text { SIZE }\end{array}$} & \multicolumn{4}{|c|}{} \\
\hline & & 1 & 5 & 1 & 5 \\
\hline & 1 & .429 & .429 & .577 & .935 \\
\hline FIFO & 3 & .669 & .669 & .577 & .935 \\
\cline { 2 - 6 } & 7 & .887 & .887 & .577 & .935 \\
\hline LRU & 3 & .676 & .676 & .577 & .935 \\
\cline { 2 - 6 } & 7 & .915 & .915 & .577 & .935 \\
\hline LFU & 3 & .746 & .746 & .577 & .935 \\
\cline { 2 - 6 } & 7 & .915 & .915 & .577 & .935 \\
\hline & 10 & .915 & .915 & .577 & .935 \\
\hline
\end{tabular}

Table 8.6 Hit ratios for tests using write-thru without validations ( 0 for PUT_CERT and PUT_CRL requests) 


\begin{tabular}{|c|c|c|c|c|c|}
\hline & & \multicolumn{3}{|c|}{ GET_CERT } & \multicolumn{2}{c|}{ GET_CRL } \\
\hline \multirow{4}{*}{$\begin{array}{c}\text { CERT } \\
\text { CACHE } \\
\text { SIZE }\end{array}$} & \multicolumn{4}{|c|}{$\begin{array}{c}\text { CRL } \\
\text { SIZE }\end{array}$} \\
\hline & & 1 & 5 & 1 & 5 \\
\hline & 1 & .443 & .443 & .577 & .935 \\
\hline FIFO & 3 & .669 & .669 & .614 & .935 \\
\cline { 2 - 6 } & 7 & .887 & .887 & .596 & .935 \\
\hline LRU & 3 & .676 & .676 & .596 & .935 \\
\cline { 2 - 6 } & 7 & .915 & .915 & .596 & .935 \\
\hline LFU & 3 & .746 & .746 & .577 & .935 \\
\cline { 2 - 6 } & 7 & .915 & .915 & .596 & .935 \\
\hline & 10 & .915 & .915 & .596 & .935 \\
\hline
\end{tabular}

Table 8.7 Hit ratios for tests using write-back without validations ( 0 for PUT_CERT and PUT_CRL requests)

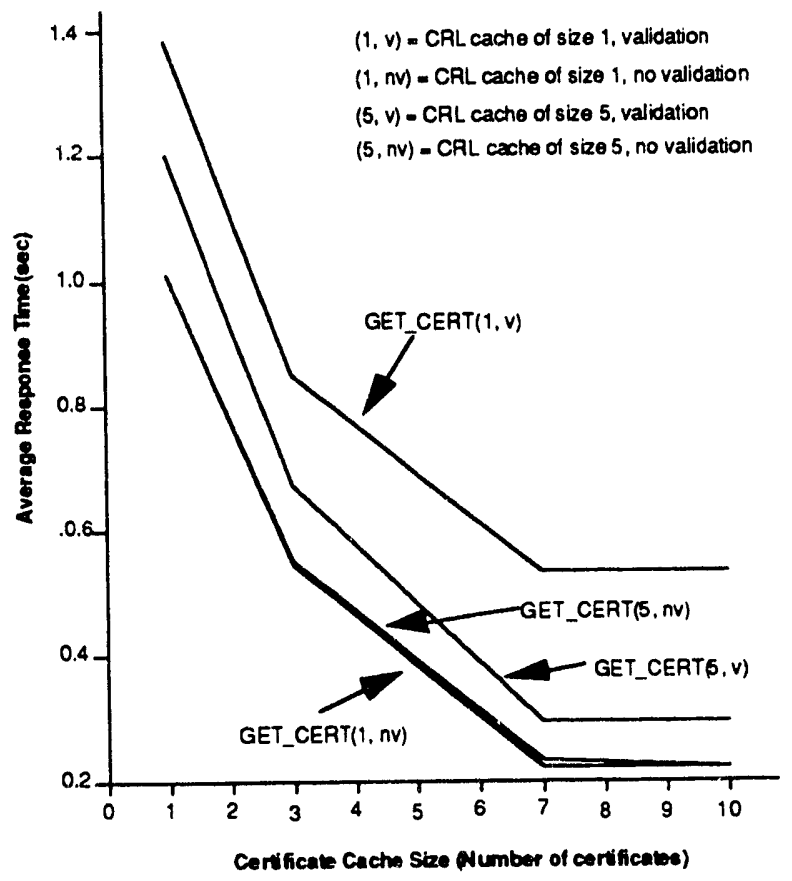

Figure 8.2 Comparison of GET_CERT for LFU using write-thru with or without validations 


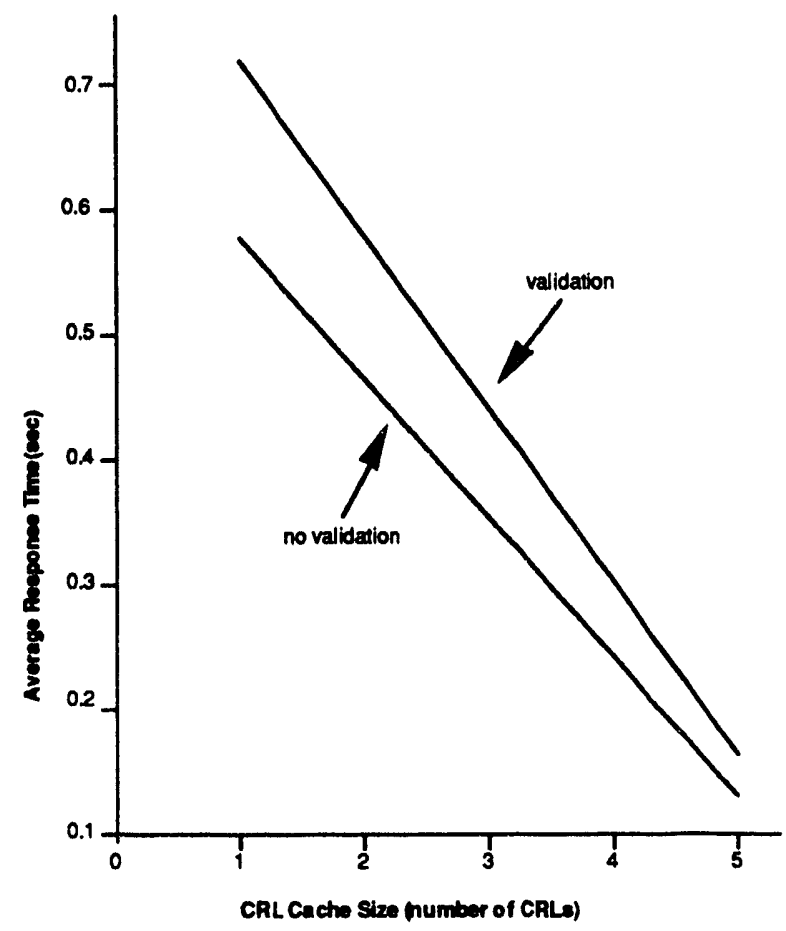

Figure 8.3 Comparison of GET_CRL for LFU using write-thru with or without validations (only certificate cache of size 7 is shown, the other sizes are pretty much identical since certificate cache sizes do not affect GET_CRL operations)

Security usually brings with it extra performance cost. Looking at the tables for the write-back strategy (Tables 8.1 and 8.5 ), we see that for small CRL caches, the response times for GET_CERT requests more than double with validation in the worst case scenarios, while for large CRL caches, the GET_CERT requests perform only slightly worse with validation. This phenomenon indicates that a major part of the added cost is a result of retrieving CRLs from the directory, rather than normal validation processing, and with appropriately large CRL caches, this added cost can be minimized. On the other hand, the GET_CRL requests performed slightly better with validation. This anomaly is attributed to the order in which TIS/PEM issues requests. With validation, when a GET_CERT request to retrieve a certificate precedes a GET_CRL request to retrieve the certificate's issuer's CRL, the GET_CERT request can bring the issuer's CRL into the cache, and thus the GET_CRL request performs better than it would have without validation. We also see that the PUT requests perform many 
times worse with validation. To summarize, validation gives GET_CRL requests a slight performance advantage, while it brings slight performance degradation to GET_CERT requests (with sufficiently large CRL caches) and significant performance cost to PUT requests. Nevertheless, since PUT requests occur much less frequently, the total added cost should be a tiny fraction of the overall times. Hence, the cache server will be able to provide reliable service through the use of validation at the cost of some extra memory and very trivial performance loss.

\subsection{Preloading}

In the previous section we ran the tests with a cold (empty) cache. Cold caches typically result in poorer performance. Thus, we want to be able to preload caches with data that are more likely to be referenced when the cache server starts up.

Based on the locality principle that recent past behavior is a good indicator of the near future, we determined that data that were referenced in the previous execution of the cache server will more likely be used again. Thus, we came up with the following cache preloading scheme, which makes use of cache logs. Periodically, the cache server writes the DNs of the cache entries to log files, and on start up, the cache server will read from log files and load the caches with certificates and CRLs whose DNs are in the $\log$ files.

To test preloading, we ran each test twice. As the result of the first run, the log file is created. The first run also ensures all the data required for startup are in the PEM archive. The disadvantage of this test scheme is that there will not be PUT requests in the second test run, since the cache server will be able to satisfy any data retrieval requests by the PEM clients, and TIS/PEM clients do not attempt to store any data if 
they are already in the database. This means that the tests with preloading will have a different test environment than those without preloading. Furthermore, there will be more GET requests in the second test run, due to a bootstrapping problem that is present in TIS/PEM when dealing with validations.

Hence, when we compare our results of performance with the preloading to those without the preloading, since the number of requests differ (there are 155 GET_CERTs and $120 \mathrm{GET}$ _CRLs in the case of preloading, as compared to 142 GET_CERTs and 109 GET_CRLs in the case of no preloading), we have to keep in mind that we are not comparing two things in identical environments. However, our measurements can still give us a feel about how much performance gain preloading provides over cold caches. Tables 8.8 through 8.11 show the results for preloading with the DEPTH algorithm, and Figures 8.4 and 8.5 compare the performance of GET_CERT and PUT_CERT using write-through with or without pre-loading. Note that in one test a pre-loading strategy actually performs worse than a cold cache. Again, this is due to different number of GET and PUT requests. In the future, when TIS/PEM fixes its bootstrapping problem, pre-loading should always perform better than a cold cache.

\begin{tabular}{|c|c|c|c|c|}
\hline & \multicolumn{3}{|c|}{ GET_CERT } & \multicolumn{2}{c|}{ GET_CRL } \\
\hline CERT & \multicolumn{4}{|c|}{ CRL } \\
CACHE \\
SIZE \\
SIZE
\end{tabular}

Table 8.8 Response times for write-thru with preloading 


\begin{tabular}{|c|c|c|c|c|}
\hline \multirow{3}{*}{$\begin{array}{c}\text { CERT } \\
\text { CACHE } \\
\text { SIZE }\end{array}$} & \multicolumn{2}{|c|}{ GET_CERT } & \multicolumn{2}{|c|}{ GET_CRL } \\
\hline & \multicolumn{4}{|c|}{$\begin{array}{l}\text { CRL } \\
\text { CACHE } \\
\text { SIZE }\end{array}$} \\
\hline & & 5 & & 5 \\
\hline 1 & 1.344 & 1.055 & .515 & .030 \\
\hline 3 & .778 & 463 & .513 & .027 \\
\hline$\overline{7}$ & .532 & .253 & .512 & .041 \\
\hline 10 & .335 & .041 & .517 & .029 \\
\hline
\end{tabular}

Table 8.9 Response times for write-back with preloading

\begin{tabular}{|c|c|c|c|c|}
\hline & \multicolumn{3}{|c|}{ GET_CERT } & \multicolumn{2}{c|}{ GET_CRL } \\
\hline CERT & \multicolumn{4}{|c|}{ CRL } \\
CACHE \\
CACHE
\end{tabular}

Table 8.10 Hit ratios for write-thru with preloading

\begin{tabular}{|c|c|c|c|c|}
\hline & GEI & & $\overline{\mathrm{GE}}$ & $\mathrm{RL}$ \\
\hline $\begin{array}{l}\text { CERT } \\
\text { CACHE } \\
\text { SIZE }\end{array}$ & \multicolumn{4}{|c|}{$\begin{array}{l}\text { CRL } \\
\text { CACHE } \\
\text { SIZE }\end{array}$} \\
\hline & 1 & 5 & 1 & 5 \\
\hline 1 & .425 & .425 & .592 & 1.0 \\
\hline$\overline{3}$ & .793 & .793 & .592 & 1.0 \\
\hline$\overline{7}$ & .883 & .883 & .592 & 1.0 \\
\hline$\overline{10}$ & 1.0 & 1.0 & .592 & 1.0 \\
\hline
\end{tabular}

Table 8.11 Hit ratios for write-back with preloading 


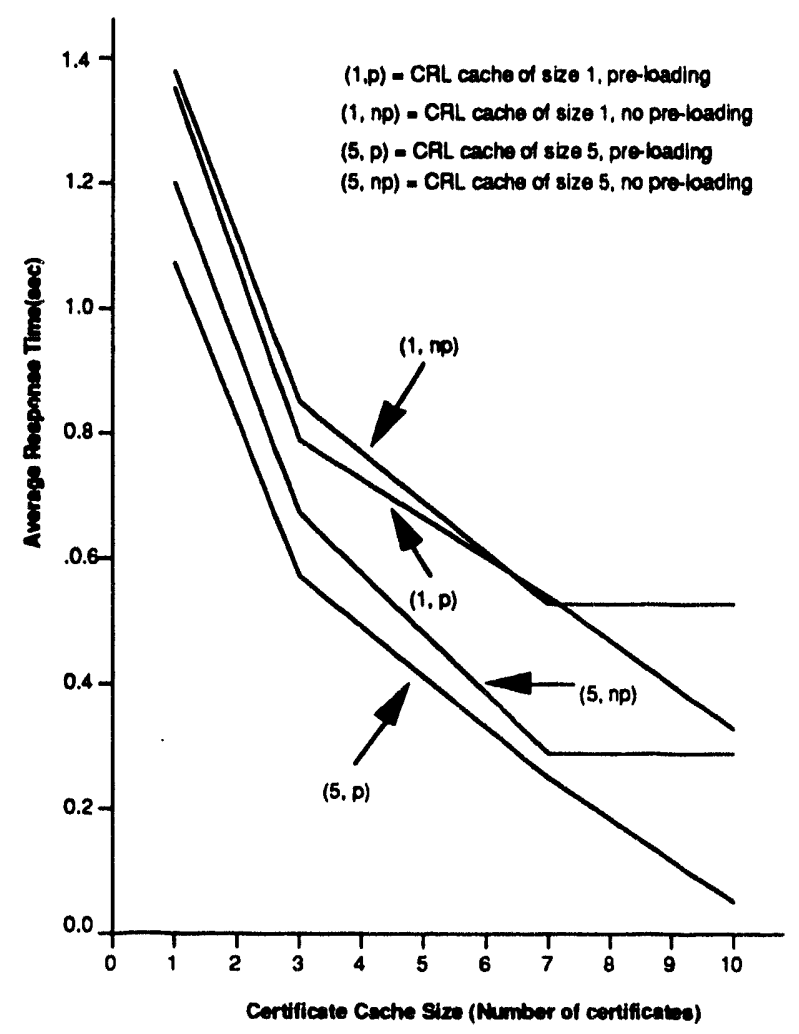

Figure 8.4 Comparison of GET_CERT for DEPTH using write-thru with or without pre-loading

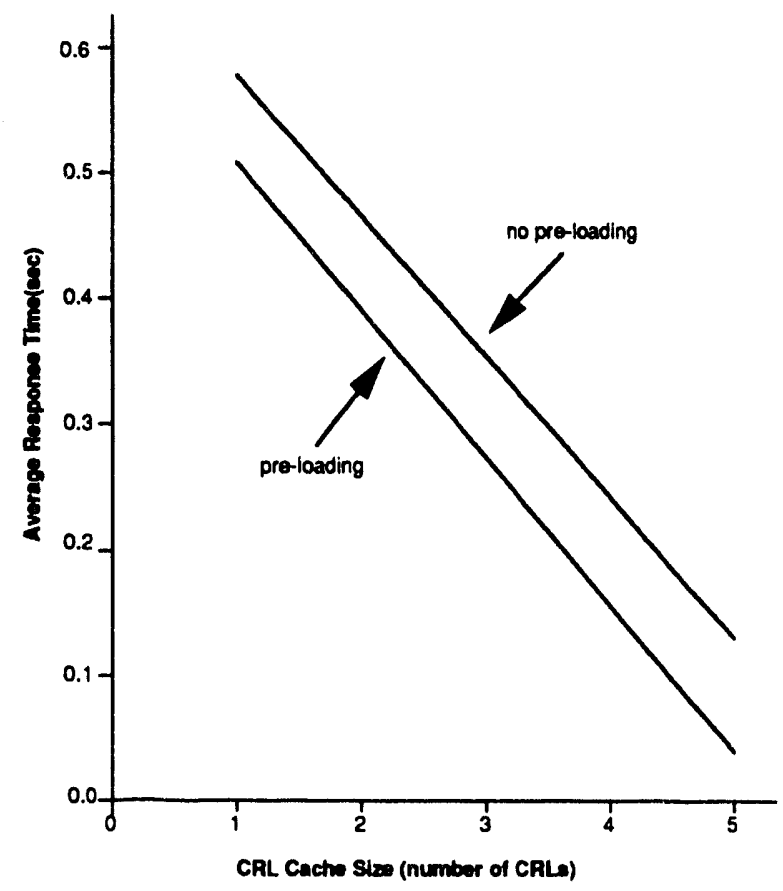

Figure 8.5 Comparison of GET_CRL for DEPTH using write-thru with or without preloading (only certificate cache of size 7 is shown, the other sizes are nearly identical since certificate cache sizes do not affect GET_CRL operations) 


\section{Persistence}

Since the modified TIS/PEM requires the existence of the cache server in order to operate, it is important that the cache server be persistent. Hence, we need to have a mechanism to ensure that the cache server is always up and running. One way to achieve this on the SUNs is to use a UNIX command "at", which executes a script at a later time. On SUNs the tasks that are scheduled to run at a later time will survive over system crashes. Our UNIX shell script searches through the active processes to see if the cache server is running, and if not, will start the cache server. The UNIX "awk" command which scans patterns is used for pattern matching.

The main script (called "persist") is shown in Figure 9.0.

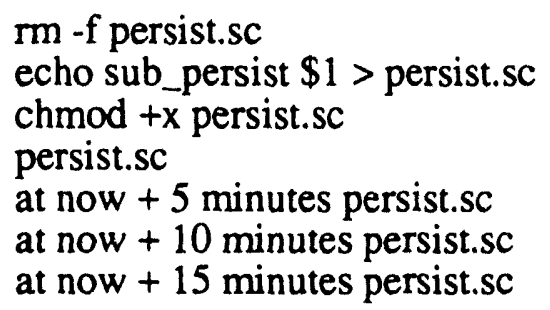

Figure 9.0 Main script - "persist"

The "persist" script expects a parameter, the name of the certificate cache server program. So the command to start the script is "persist <cache server program name>." "Persist" generates another script "persist.sc," and executes "persist.sc" four times, each of them 5 minutes apart. Five minutes are short enough so that there is a check to see if the cache server is running every 5 minutes. Four tasks will span over a period of 20 minutes, so that some tasks can survive over system crashes if the system is brought up 
within 20 minutes. It should be easy to write other scripts that will handle system crashes of over 20 minutes, such as running "persist" at one day intervals, etc.

Suppose the cache server program is named "certificate_cache_server," then "persist.sc" contains the line shown in Figure 9.1.

sub_persist certificate_cache_server

Figure 9.1 Script "persist.sc"

"Sub_persist" is a script containing the lines shown in Figure 9.2.

ps $-\mathrm{x} \mid$ awk $-\mathrm{f}$ a.persist proc $=\$ 1$

chmod $+x$ aux_persist

aux_persist

rm aux_persist

Figure 9.2 Script "sub_persist"

"Sub_persist" uses "awk" to generate yet another script "aux_persist," which contains the line shown in Figure 9.3 if the cache server is running.

$$
\text { at now }+5 \text { minutes persist.sc }
$$

Figure 9.3 "Aux_persist" file generated when the cache server is active

"Aux_persist" will contain the lines shown in Figure 9.4 if the cache server is not currently active. 
certificate_cache_server $>/ \mathrm{dev} /$ null $\&$ at now +5 minutes persist.sc

Figure 9.4 "aux_persist" file generated when the cache server is not active

Finally, the "awk" input program "a.persist" contains the lines shown in Figure 9.5.

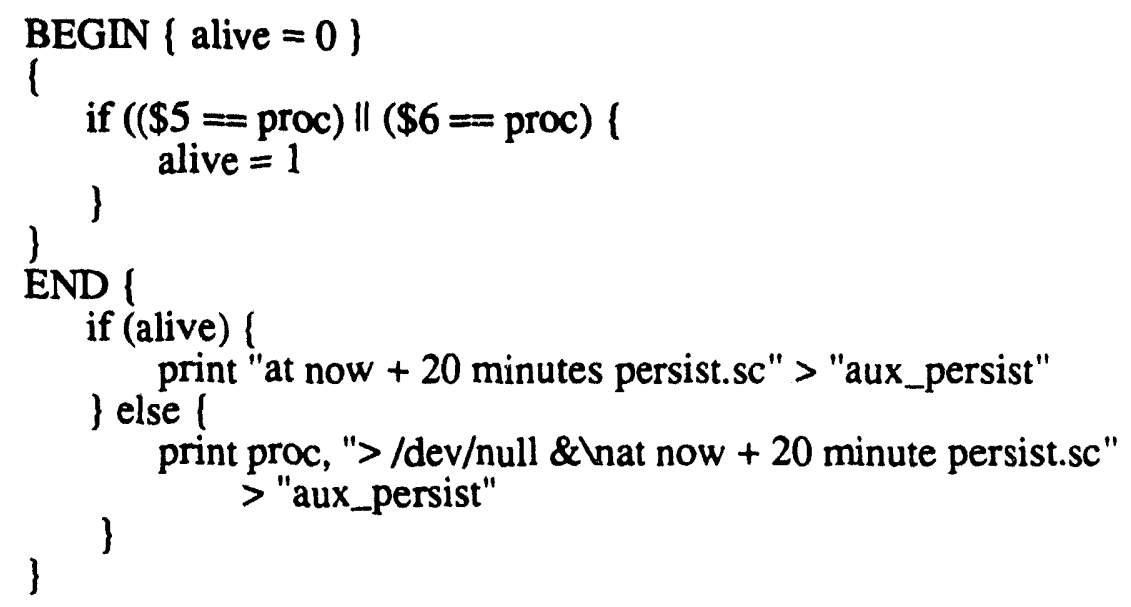

Figure 9.5 The "awk" input program - "a.persist" 


\section{Conclusions and Future Work}

In this project we have designed a certificate cache server that helps provide effective sharing of public key certificates among PEM clients at different sites. While designing the server we have addressed the security problem of denial-of-service attacks by providing validations of certificates and CRLs in the cache server.

Our cache server has promise in providing a smooth transition for PEM to migrate from the current environment to the future environment when X.500 is ubiquitous. By maintaining the existing push-model approach in our design, we ensure that the modified PEM implementation can still operate during the transition period. Introducing the pull-model approach into the design allows the retrieving of certificates from the X.500 directory, which is desired when certificates can be easily distributed through the X.500 directory.

As we have seen, the performance associated with the cache server approach is several times worse than that with the original push model. However, the majority of cache server requests perform rather well. The poor average response time is caused by few requests that involved directory operations. Hence, one way to bring the performance of the cache server approach closer to that of the push model approach is to place the backing store on a file system local to the cache server. Accesses to the backing store by the cache server will then be much faster. This approach should be able to significantly improve the performance of the cache server .

Another possible improvement is that is that all certificates on a certification path have to be validated by TIS/PEM clients due to the lack of a secure channel between the 
TIS/PEM clients and the cache server. If a secure channel can be maintained between the cache server and TIS/PEM clients, then TIS/PEM clients do not have to perform validations, thus saving a significant number of data retrievals. The secure channel need not provide privacy. It only needs to guarantee the integrity of the data in transit. 


\section{References}

[Bran92] Branstetter, M., Guse, J., and Nessett, D., "ELROS - An Embedded Language for Remote Operations Service," Proc. 1992 IFIP International Conference on Upper Layer Protocols, Architectures and Applications, Vancouver, Canada, May 25-29, 1992.

[Carr87] Carroll, J., Computer Security, Second Edition, Butterworth-Heinemann, Stoneham, Massachusetts, 1987.

[Galv92] Galvin, J. and Balenson, D., "Security Issues of a UNIX PEM Implementation, " TIS Technical Report 468D, Trusted Information Systems, Inc., Glenwood, Maryland, February 11, 1993.

[Hous92] Housley, R. and Hollenbeck, S., "Privacy Enhanced Mail: An Implementation Summary," independent study project, George Mason University, Fairfax, Virginia, May, 1992.

[Ishi93] Ishida H. and Landweber L., "Internetworking Introduction," Communications of the ACM, Vol 36, No. 8, pp. 28-30, August, 1993.

[ISOD92] "The ISO Development Environment: User's Manual," June, 1992.

[Kent93] Kent, S., "Internet Privacy Enhanced Mail, " Communications of the $A C M$, Vol 36, No. 8, pp. 48-60, August, 1993.

[RFC1421] Linn, J., "Privacy Enhancement for Internet Electronic Mail: Part I: Message Encryption and Authentication Procedures," Network Working Group, RFC-1421, February, 1993.

[RFC1422] Kent, S., "Privacy Enhancement for Internet Electronic Mail: Part II: Certificate-Based Key Management," Network Working Group, RFC1422, February, 1993.

[RFC1423] Balenson, D., "Privacy Enhancement for Internet Electronic Mail: Part III: Algorithms, Modes, and Identifiers," Network Working Group, RFC1423, February, 1993.

[RFC1424] Kaliski, B., "Privacy Enhancement for Internet Electronic Mail: Part IV: Key Certification and Related Services" Network Working Group, RFC1424, February, 1993.

[Rose92] Rose, M., The I ittle Black Boook: Mail Bonding with OSI Directory Services, Prentice-Hall, Inc. Englewood Cliffs, New Jersey, 1992.

[Smit82] Smith, A., "Cache Memories, " ACM Computing Surveys, Vol. 14, No. 3, pp. 473-530, September, 1982.

[Tay193] Taylor, M., "Implementing Privacy Enhanced Mail on VMS, " Proc. PSRG Workshop on Network and Distributed System Security, San Diego, California, February 11-12, 1993, pp. 63-68. 
[X.500-88] "The Directory - Overview of Concepts, Models, and Service," International Telegraph and Telephone Consultative Committee, December, 1988. Recommendation X.500.

[X.509-88] "The Directory - Authentication Framework," International Telegraph and Telephone Consultative Committee, December, 1988. Recommendation X.509. 


\section{Appendix A: Flow Diagrams for the Cache Server Operations}

The following sections contain flow diagrams that shows the actions taken by the cache server when receiving different requests. In addition, since storing a certificate or CRL into the caches goes through similar processing, the part of the flowchart that illustrates the process of storing of a certificate or a CRL into a cache is displayed in section A.5. 


\section{A.1. GET CERT}

On receiving a GET_CERT request:

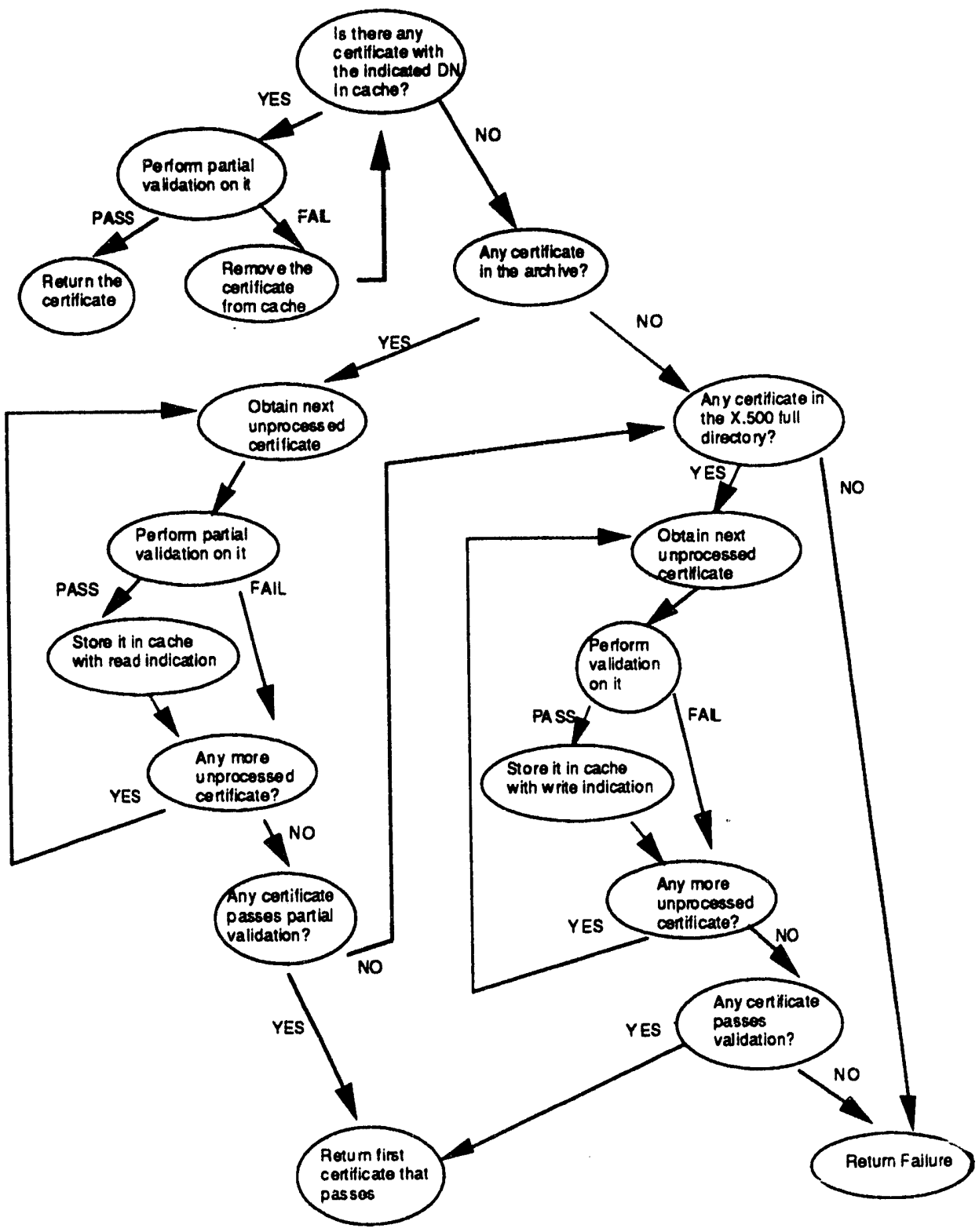

Figure A.1 Flowchart of cache server processing on receiving a GET_CERT request 
66

A.2. PUT CERT

On receiving a PUT_CERT request:

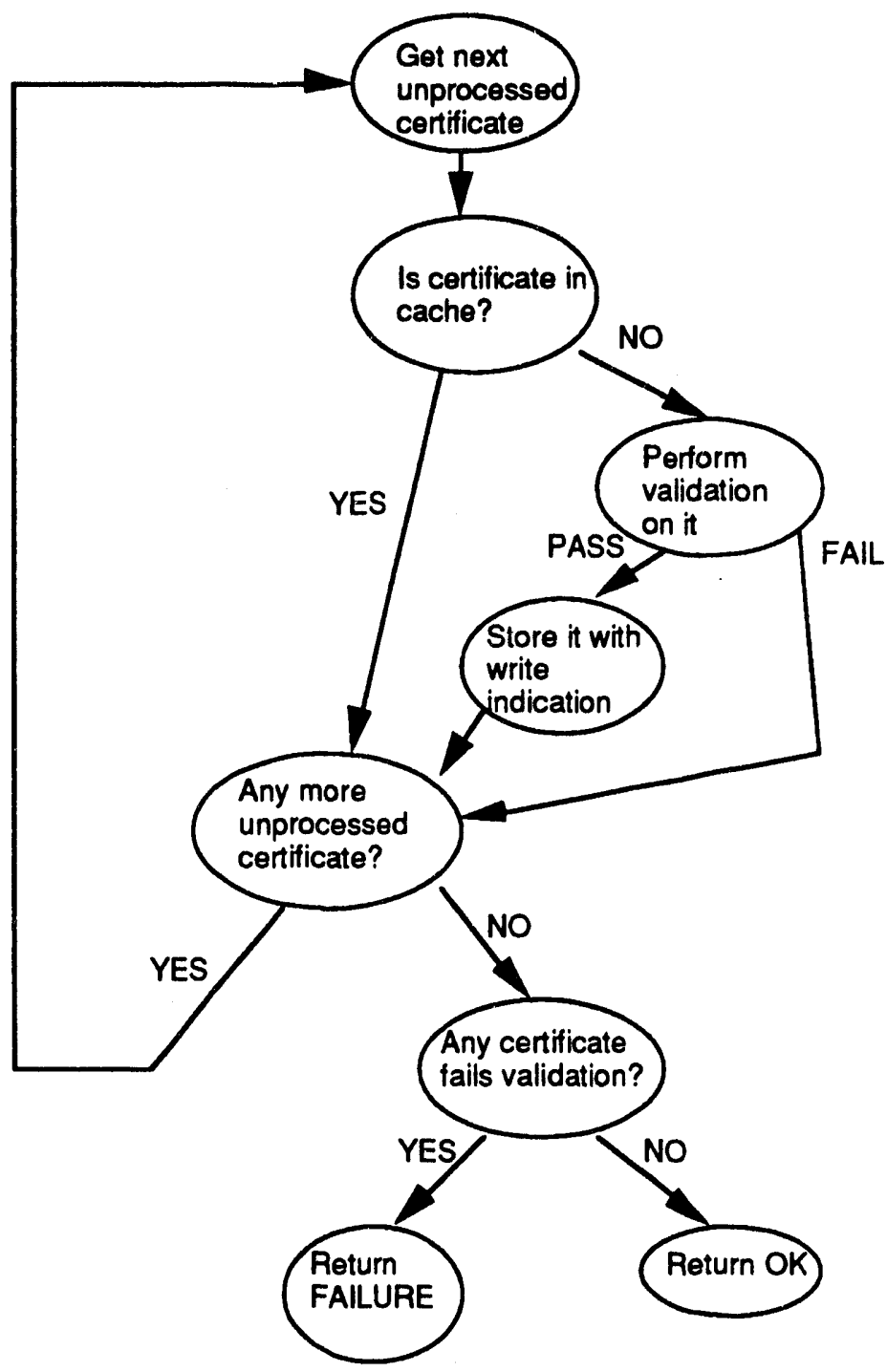

Figure A.2 Flowchart of cache server processing on receiving a PUT_CERT request 


\section{A.3. GET CRL}

On receiving a GET_CRL request:

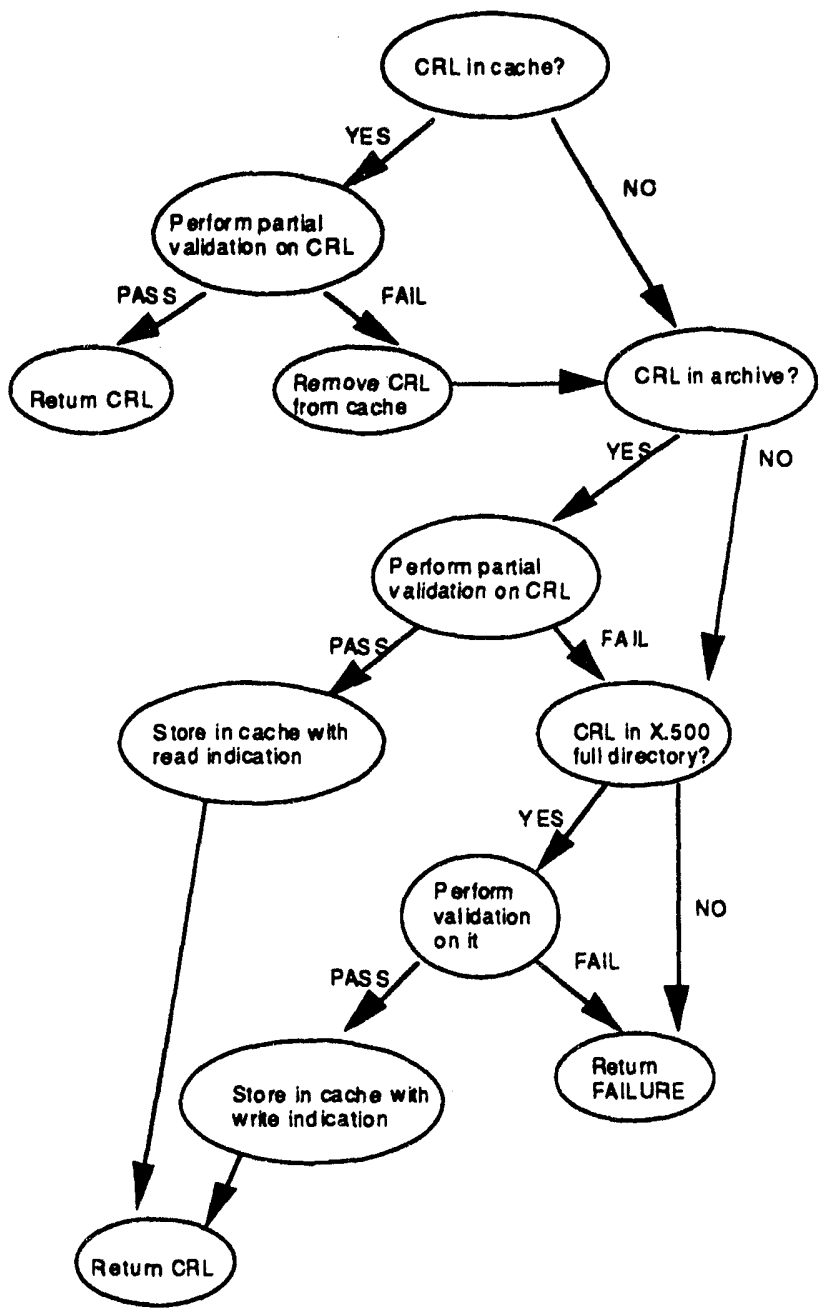

Figure A.3 Flowchart of cache server processing on receiving a GET_CRL request 


\section{A.4. PUT CRL}

On receiving a PUT_CRL request:

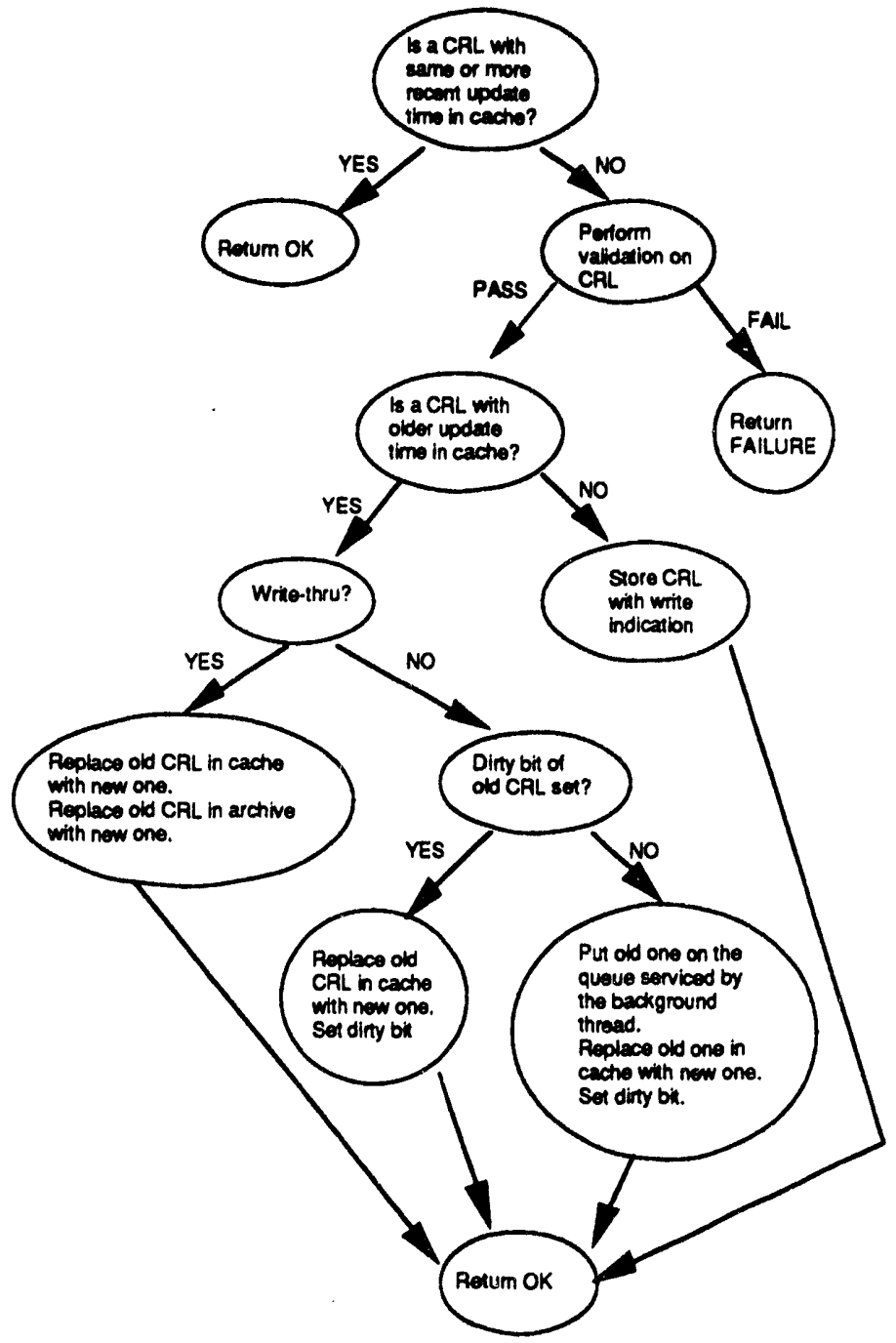

Figure A.4 Flowchart of cache server processing on receiving a PUT_CRL request 


\section{A.5. Storing a certificate or CRL into the caches}

The following flowchart shows the processing of the cache server when it stores a certificate or $\mathrm{CRL}$ into the appropriate cache. On storing a certificate or CRL into a cache, there is a notion of storing with read or write indication, which is used to indicate whether the data being stored should also be written to the archive or not. 


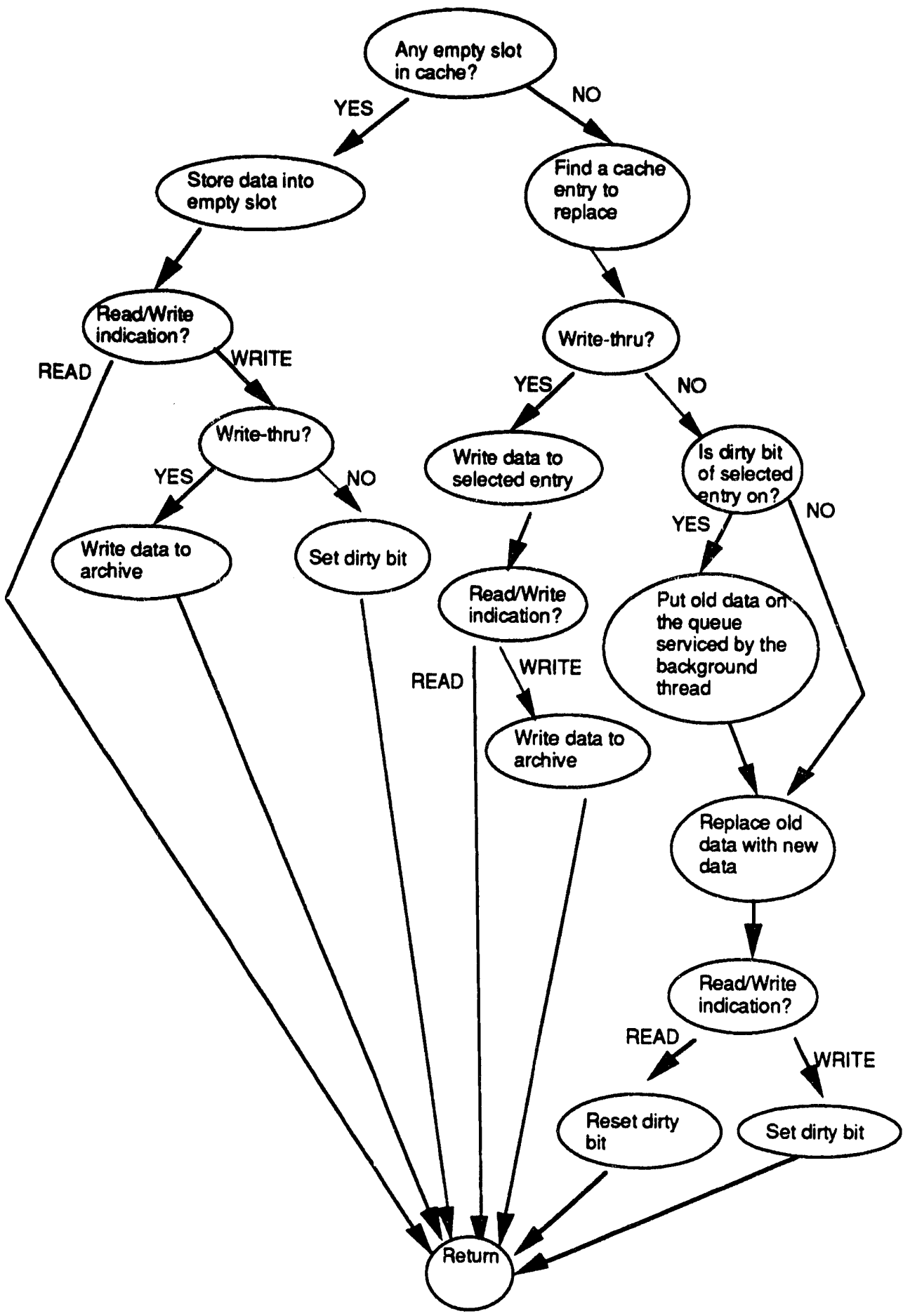

Figure A.5 Flowchart of cache server processing on storing a certificate or a CRL into the caches 

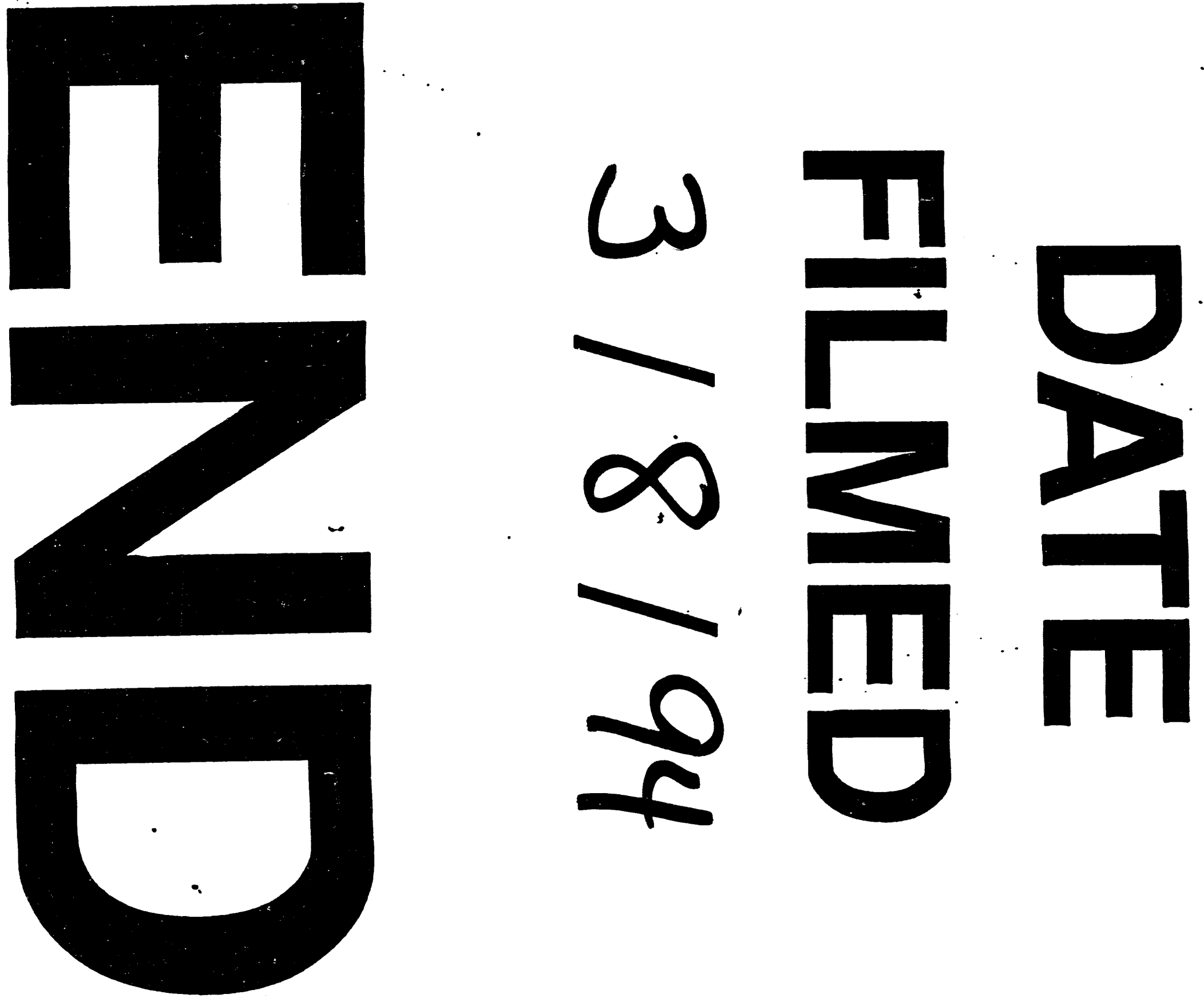
